\title{
Adverse effects of immune-checkpoint inhibitors: epidemiology, management and surveillance
}

Filipe Martins ${ }^{1,2}$, Latifyan Sofiya ${ }^{3}$, Gerasimos P. Sykiotis ${ }^{4}$, Faiza Lamine ${ }^{4}$, Michel Maillard ${ }^{5,6}$, Montserrat Fraga ${ }^{5}$, Keyvan Shabafrouz ${ }^{3}$, Camillo Ribi ${ }^{7}$, Anne Cairoli ${ }^{1}$, Yan Guex-Crosier (1) ${ }^{8}$, Thierry Kuntzer ${ }^{9}{ }^{9}$, Olivier Michielin ${ }^{3}$, Solange Peters ${ }^{3}$, Georges Coukos ${ }^{3}, 10$, Francois Spertini ${ }^{7}$, John A. Thompson ${ }^{11,12}$ and Michel Obeid ${ }^{7,13,14 *}$

Abstract | Immune-checkpoint inhibitors (ICls), including anti-cytotoxic T lymphocyte antigen 4 (CTLA-4), anti-programmed cell death 1 (PD-1) and anti-programmed cell death 1 ligand 1 (PD-L1) antibodies, are arguably the most important development in cancer therapy over the past decade. The indications for these agents continue to expand across malignancies and disease settings, thus reshaping many of the previous standard-of-care approaches and bringing new hope to patients. One of the costs of these advances is the emergence of a new spectrum of immune-related adverse events (irAEs), which are often distinctly different from the classical chemotherapy-related toxicities. Owing to the growing use of ICls in oncology, clinicians will increasingly be confronted with common but also rare irAEs; hence, awareness needs to be raised regarding the clinical presentation, diagnosis and management of these toxicities. In this Review, we provide an overview of the various types of irAEs that have emerged to date. We discuss the epidemiology of these events and their kinetics, risk factors, subtypes and pathophysiology, as well as new insights regarding screening and surveillance strategies. We also highlight the most important aspects of the management of irAEs.

Monoclonal antibodies targeting immune checkpoints are able to restore antitumour immunity, thus reversing immune escape or evasion and promoting tumour cell death. Such antibodies include those targeting the cytotoxic T lymphocyte antigen 4 (CTLA-4)-CD28 and programmed cell death 1 (PD-1)-programmed cell death 1 ligand 1 (PD-L1) axes. CTLA-4 is a receptor expressed on the surface of $\mathrm{T}$ cells that modulates CD28 co-stimulatory signalling by competing for its activating ligands (CD80 and CD86) that are expressed on the surface of antigen-presenting cells during the early phase of the immune response, consequently impairing $\mathrm{T}$ cell activation. Activation of CTLA- 4 inhibits the NF- $\mathrm{\kappa B}$ signalling pathway, leading to impaired IL-2 production ${ }^{1,2}$. CTLA-4 is also constitutively expressed on regulatory $\mathrm{T}\left(\mathrm{T}_{\text {reg }}\right)$ cells, which promote immunosuppression in the tumour microenvironment (TME) ${ }^{3}$. PD- 1 is also a cell surface receptor, which recognizes and binds to the endogenous ligands PD-L1 and PD-L2. This receptor is a member of the immunoglobulin superfamily and is expressed on T cells and B cells but also on cells involved in innate immunity, such as natural killer cells and myeloid cells ${ }^{4}$. Activation of either or both of these receptors has an inhibitory effect on the T cell response, thus, in the absence of a malignancy, inducing immune tolerance and preventing autoimmunity ${ }^{5}$. However, both immune checkpoints can also be hijacked by cancer cells in order to develop a microenvironment that is tolerant of tumour growth ${ }^{6}$.

The introduction of ipilimumab (an anti-CTLA-4 antibody) into clinical practice transformed the prognosis of patients with advanced-stage melanoma by enabling a subset of patients with this previously universally terminal disease (20-30\%) to achieve long-term remissions ${ }^{7}$. Following this initial success, anti-PD-1 and anti-PD-L1 antibodies entered the clinic, resulting in dramatic improvements in the prognosis of patients with melanoma or several other advanced-stage malignancies. Since 2015, anti-CTLA-4 and anti-PD-1 antibodies have shown tremendous efficacy when used in combination, with increased response rates in patients with advanced-stage melanoma ${ }^{8}$, renal cell carcinoma (RCC) ${ }^{9}$, microsatellite instability (MSI)-high cancers ${ }^{10}$, small-cell lung cancer (SCLC) ${ }^{11}$ and non-small-cell lung cancer 


\section{Key points}

- The frequency of immune-related adverse events (irAEs) is dependent on the agents used, exposure time and the administered dose but also on the patient's intrinsic risk factors; conversely, the timing of appearance is often dictated by the affected organ systems.

- High-risk patients receiving immune-checkpoint inhibitors (ICls) should be regularly monitored for treatment-related complications by specialized multidisciplinary teams, ideally using a personalized surveillance strategy.

- The application of formal contraindications to the use of ICls among patients with a high risk of irAEs is not supported by well-founded scientific evidence.

- In patients with severe and/or steroid-refractory irAEs, a biopsy sample should be obtained and investigated for infiltrating immune cells in order to enable the selection of novel biological agents targeting key inflammatory mediators.

(NSCLC) $)^{12,13}$ compared with either agent as monotherapy, albeit at the cost of increased toxicities. These toxicities are mainly considered immune-related adverse events (irAEs), meaning that they are off-target effects of an excessively activated immune system. As a consequence of the improved survival provided by immunecheckpoint inhibitors (ICIs), clinicians are increasingly confronted with patients with irAEs, which often require new skills to successfully diagnose and manage.

The frequency of irAEs is mainly dependent on the agents used but also on the specific characteristics of individual patients (TABLE 1). The incidence of fatal ICIassociated adverse events is estimated to be between $0.3 \%$ and $1.3 \%{ }^{14}$ (TABLE 2). This risk remains lower than that associated with conventional treatments: for example, $\sim 0.9 \%$ with platinum-doublet chemotherapy ${ }^{15}$, $\sim 15 \%$ with allogeneic haematopoietic stem cell transplantation ${ }^{16}$ and $0-4 \%$ with targeted therapies, such as VEGF-targeted agents or tyrosine kinase inhibitors (TKIs) ${ }^{9}$. Regardless of the ICI used, toxicities with fatal outcomes tend to occur early in the course of treatment and evolve rapidly, especially in patients receiving combinations of agents. The median time to the onset of a fatal toxic event is $\sim 14.5$ days for ICI combinations, whereas the onset of such events tends to be delayed to 40 days in patients receiving ICI monotherapies $(P<0.001)^{13}$. The spectrum of fatal irAEs differs widely between regimens, as demonstrated in a meta-analysis

\footnotetext{
Author addresses

'Service et Laboratoire Central d'Hématologie, Département d'Oncologie, Centre Hospitalier Universitaire Vaudois (CHUV), Lausanne, Switzerland.

${ }^{2}$ School of Life Sciences, Ecole Polytechnique Fédérale de Lausanne (EPFL), Lausanne, Switzerland.

${ }^{3}$ Département d'Oncologie, CHUV, Lausanne, Switzerland.

${ }^{4}$ Service d'Endocrinologie, Diabétologie et Métabolisme, CHUV, Lausanne, Switzerland.

${ }^{5}$ Service de Gastro-entérologie et Hépatologie, CHUV, Lausanne, Switzerland.

${ }^{6}$ Crohn's and Colitis Center Lausanne, Lausanne, Switzerland.

${ }^{7}$ Service Immunologie et Allergie, CHUV, Lausanne, Switzerland.

${ }^{8}$ Hôpital Ophtalmique Jules Gonin - Fondation Asile des Aveugles, CHUV, Lausanne,

Switzerland.

${ }^{9}$ Service de Neurologie, CHUV, Lausanne, Switzerland.

${ }^{10}$ Ludwig Institute for Cancer Research, Epalinges, Switzerland.

${ }^{11}$ Fred Hutchinson Cancer Research Center, Seattle, WA, USA.

${ }^{12}$ National Cancer Institute, NIH, Bethesda, MD, USA.

${ }^{13}$ Faculté de Médecine Pitié-Salpêtrière, Centre Hospitalo-Universitaire Pitié-Salpêtrière, Université Pierre et Marie Curie, Paris, France.

${ }^{14}$ Centre d'Immunothérapie et de Vaccinologie, CHUV, Lausanne, Switzerland.
}

published in December 2018 (REF. $\left.{ }^{13}\right)$. In this analysis, colitis was the most frequent cause of death as an irAE in patients receiving anti-CTLA-4 antibodies (135 (70\%) of 193 deaths), whereas fatalities in patients receiving antiPD-1 or anti-PD-L1 antibodies were mainly attributed to pneumonitis (115 (35\%) of 333), hepatitis (75 (22\%) of 333$)$ and neurotoxic effects $(50(15 \%)$ of 333$)$. In patients receiving combination therapies, ICI-related deaths were mainly attributed to colitis (32 (37\%) of 87) or myocarditis (22 (25\%) of 87$)$. Of note, patients who develop myocarditis as an irAE have the highest fatality rate (52 (39.7\%) of 131 events reported) across all treatment groups ${ }^{14}$.

A thorough understanding of irAEs, including the underlying pathogenesis, kinetics of appearance and clinical presentation, will not only help clinicians to manage these events more effectively but also enable assessments of the safety of treatment resumption after irAE resolution. Rare forms of irAE are increasingly being reported in the medical literature, and clinicians must take into consideration the heterogeneous clinical presentations of patients with these events and the broad spectrum of affected organs. This suggestion underlines the fact that the management of patients with irAEs often goes beyond the field of oncology and frequently demands a multidisciplinary approach. New monoclonal antibodies targeting other immune checkpoints are currently being tested in clinical trials and might soon further enrich the therapeutic arsenal. Therefore, highlighting what is already known about the demographics, kinetics and pathogenesis of irAEs related to anti-CTLA-4, anti-PD-1 and anti-PD-L1 antibodies will be useful in revealing current unmet needs and possibly in preparing oncologists for the challenges created by the introduction of new ICIs with novel mechanisms of action. No widely validated strategies for the surveillance of irAEs are currently available, and a greater understanding of the variations in the propensity of patients to develop irAEs is clearly required. A general consensus exists, however, that the optimal management of irAEs relies primarily on early recognition in order to limit the need for treatment interruptions, preserve quality of life and avoid or minimize the risk of rare fatal outcomes.

\section{Incidence and kinetics of irAEs Anti-CTLA-4 antibodies}

Ipilimumab was the first ICI to demonstrate a survival benefit in patients with metastatic melanoma. IrAEs of any grade can occur in up to $60 \%$ of patients treated with ipilimumab, and $10-30 \%$ of these are typically considered serious (defined as grade 3-4 according to the National Cancer Institute's Common Terminology Criteria for Adverse Events (CTCAE)). In a phase III trial comparing the efficacy of a $10 \mathrm{mg} / \mathrm{kg}$ dose of ipilimumab with that of a $3 \mathrm{mg} / \mathrm{kg}$ dose administered on the same schedule in patients with previously treated advanced-stage melanoma, patients in the high-dose ipilimumab group had an increased prevalence of grade $\geq 3$ adverse events ( $37 \%$ versus $18 \%$; TABLE 1). This finding suggests that the risk of irAEs in patients receiving ipilimumab is dose-dependent ${ }^{17,18}$. In a cohort of patients with high-risk stage III melanoma, patients received 
Table 1 | Frequencies of treatment-related irAEs in selected cohorts

\begin{tabular}{|c|c|c|c|c|c|c|c|c|c|}
\hline \multicolumn{2}{|l|}{ Study details } & \multicolumn{8}{|c|}{ Any-grade adverse events (grade $\geq 3$ adverse events) } \\
\hline Study & Dose $(n)$ & Diarrhoea & Colitis & Pulmonary & Rash & Neurological & Endocrinopathy & Hepatic & Renal \\
\hline \multicolumn{10}{|l|}{ Ipilimumab } \\
\hline Hodi et al. ${ }^{166}$ & $\begin{array}{l}3 \mathrm{mg} / \mathrm{kg}, \\
\text { 3-weekly (131) }\end{array}$ & $27.5 \%(4.6 \%)$ & $7.6 \%(5.3 \%)$ & - & $19.1 \%(0.8 \%)$ & - & $7.6 \%(3.8 \%)$ & $3.8 \%(0 \%)$ & - \\
\hline $\begin{array}{l}\text { CheckMate } \\
066\left(\text { REF. }^{21}\right)\end{array}$ & $\begin{array}{l}3 \mathrm{mg} / \mathrm{kg}, \\
\text { 2-weekly (206) }\end{array}$ & $16 \%(1 \%)$ & $1 \%(0.5 \%)$ & $1.5 \%(0 \%)$ & $15 \%(0.5 \%)$ & - & $7.3 \%(1 \%)$ & $3.4 \%(1.5 \%)$ & $\begin{array}{l}1.9 \% \\
(0.5 \%)\end{array}$ \\
\hline $\begin{array}{l}\text { CheckMate } \\
057\left(\text { REF. }^{167} \text { ) }\right.\end{array}$ & $\begin{array}{l}3 \mathrm{mg} / \mathrm{kg}, \\
\text { 2-weekly (287) }\end{array}$ & $8 \%(1 \%)$ & $1 \%(0.3 \%)$ & $4.9 \%(1.4 \%)$ & $9 \%(3.5 \%)$ & $0.3 \%(0.3 \%)^{\mathrm{a}}$ & $10.5 \%(0 \%)$ & $10.8 \%(1.4 \%)$ & $2 \%(0 \%)$ \\
\hline \multicolumn{10}{|l|}{ Pembrolizumab } \\
\hline $\begin{array}{l}\text { KEYNOTE-010 } \\
\left(\text { REF. }^{146}\right)\end{array}$ & $\begin{array}{l}2 \mathrm{mg} / \mathrm{kg}, \\
\text { 3-weekly (339) }\end{array}$ & $7 \%(1 \%)$ & $1 \%(1 \%)$ & $5 \%(2 \%)$ & $9 \%(0.3 \%)$ & - & $15 \%(1 \%)$ & $0.3 \%(0.3 \%)$ & - \\
\hline $\begin{array}{l}\text { KEYNOTE-054 } \\
\left(\text { REF. }^{99}\right)\end{array}$ & $\begin{array}{l}200 \text { mg, } \\
\text { 3-weekly (509) }\end{array}$ & $19.1 \%(0.8 \%)$ & $3.7 \%(2 \%)$ & $4.7 \% \mathrm{~b}(0.8 \%)$ & $16.1 \%(0.2 \%)$ & - & $23.4 \%(1.8 \%)$ & $1.8 \%(1.4 \%)$ & $\begin{array}{l}0.4 \% \\
(0.4 \%)\end{array}$ \\
\hline \multicolumn{10}{|c|}{ Ipilimumab plus nivolumab } \\
\hline $\begin{array}{l}\text { CheckMate } \\
067\left(\text { REF. }^{168} \text { ) }\right.\end{array}$ & $\begin{array}{l}3 \mathrm{mg} / \mathrm{kg} \\
\text { ipilimumab } \\
\text { plus } 1 \mathrm{mg} / \mathrm{kg} \\
\text { nivolumab, } \\
\text { 3-weekly (313) }\end{array}$ & $45 \%(9 \%)$ & $13 \%(8 \%)$ & $7 \%(1 \%)$ & $30 \%(3 \%)$ & - & $34 \%(6 \%)$ & $33 \%(20 \%)$ & $7 \%(2 \%)$ \\
\hline $\begin{array}{l}\text { CheckMate } \\
214\left(\text { REF. }^{9}\right)\end{array}$ & $\begin{array}{l}1 \mathrm{mg} / \mathrm{kg} \\
\text { ipilimumab } \\
\text { plus } 3 \mathrm{mg} / \mathrm{kg} \\
\text { nivolumab, } \\
\text { 3-weekly (547) }\end{array}$ & $27 \%(4 \%)$ & - & - & $22 \%(1 \%)$ & - & $16 \%(0.4 \%)^{c}$ & - & - \\
\hline \multicolumn{10}{|l|}{ Avelumab } \\
\hline $\begin{array}{l}\text { JAVELIN Solid } \\
\text { Tumour }{ }^{169}\end{array}$ & $\begin{array}{l}10 \mathrm{mg} / \mathrm{kg}, \\
\text { 2-weekly (184) }\end{array}$ & $7 \%(0 \%)$ & - & $1 \%(1 \%)$ & - & $1 \%(1 \%)^{d}$ & $7 \%(0 \%)$ & $1.6 \%(1.1 \%)^{e}$ & - \\
\hline $\begin{array}{l}\text { JAVELIN } \\
\text { Merkel } 200 \\
\left(\text { REF. }^{170}\right)\end{array}$ & $\begin{array}{l}10 \mathrm{mg} / \mathrm{kg}, \\
\text { 2-weekly (88) }\end{array}$ & $10 \%(0 \%)$ & - & $1 \%(0 \%)$ & $13 \%(0 \%)$ & - & $7 \%(0 \%)$ & $6.8 \%(2 \%)^{\mathrm{e}}$ & $1 \%(0 \%)$ \\
\hline \multicolumn{10}{|l|}{ Atezolizumab } \\
\hline OAK $^{171}$ & $\begin{array}{l}\text { 1,200 mg, } \\
\text { 3-weekly (609) }\end{array}$ & $15.4 \%(0.7 \%)$ & $0.3 \%(0 \%)$ & $1 \%(0.7 \%)$ & - & - & - & $0.3 \%(0.3 \%)$ & - \\
\hline \multicolumn{10}{|l|}{ Durvalumab } \\
\hline ATLANTIC $^{172}$ & $\begin{array}{l}10 \mathrm{mg} / \mathrm{kg}, \\
\text { 2-weekly (444) }\end{array}$ & $0.7 \%(0.2 \%)^{f}$ & $0.4 \%(0 \%)^{f}$ & $2 \%(0.7 \%)^{f}$ & $0.7 \%(0.2 \%)^{f}$ & - & $10.1 \%(0.5 \%)^{f}$ & $0.7 \%(0.7 \%)^{f}$ & - \\
\hline
\end{tabular}

irAE, immune-related adverse event. ${ }^{\mathrm{a}}$ Encephalitis. ${ }^{\mathrm{b}} \mathrm{S} a r c o i d o s i s ~ i n ~ 1.4 \% .{ }^{\mathrm{c}} \mathrm{All}$ hypothyroidism. ${ }^{\mathrm{d}}$ Monoplegia. ${ }^{\mathrm{e}}$ Transaminase increase (treatment related or unrelated). ${ }^{\dagger} A d v e r s e$ events of special interest that required the use of systemic steroids, other immunosuppressants or endocrine therapy, and with no clear other cause; therefore, the percentages reported here probably do not reflect the true rate of irAEs of any grade because any irAEs events that were not managed with such treatments, for example, would have been excluded.

$10 \mathrm{mg} / \mathrm{kg}$ doses of adjuvant ipilimumab on a 3-weekly and then 3-monthly basis for up to 3 years ${ }^{17}$. In this study, $54.1 \%$ of patients had grade $\geq 3$ treatment-related adverse events, including immune-related deaths in 5 patients (1.1\%), highlighting that long-term treatment with highdose anti-CTLA-4 antibodies is associated with a high risk of irAEs.
The majority of grade $\geq 3$ irAEs occur within 8-12 weeks of commencing treatment, with diarrhoea and/or colitis being the most common (occurring in $\sim 20 \%$ of patients) and skin rash usually having the earliest onset (FIG. 1a). Other, less-frequent toxicities (occurring in 3-20\% of patients) include pruritus, hepatitis and endocrinopathies, such as hypophysitis and thyroiditis. 
Table 2 | Frequencies of treatment-related deaths in selected cohorts

\begin{tabular}{|c|c|c|c|}
\hline \multicolumn{2}{|l|}{ Study details } & \multicolumn{2}{|c|}{ Treatment-related deaths } \\
\hline Study & Dose (n) & Deaths (\%) & Causes of death \\
\hline \multicolumn{4}{|l|}{ Ipilimumab } \\
\hline EORTC $18071\left(\right.$ REF. $\left.^{17}\right)$ & 10 mg/kg, 3-weekly (471) & $5(1.1)$ & $\begin{array}{l}\text { Colitis in three patients, myocarditis } \\
\text { in one patient and multiple organ } \\
\text { failure associated with Guillain-Barré } \\
\text { syndrome in one patient }\end{array}$ \\
\hline Hodi et al. ${ }^{166}$ & 3 mg/kg, 3-weekly (131) & $2(1.4)$ & $\begin{array}{l}\text { Colitis in one patient and liver failure } \\
\text { in one patient }\end{array}$ \\
\hline \multicolumn{4}{|l|}{ Nivolumab } \\
\hline CheckMate 066 (REF. ${ }^{21}$ ) & 3 mg/kg, 2-weekly (206) & 0 & - \\
\hline CheckMate 057 (REF. ${ }^{167}$ ) & 3 mg/kg, 2-weekly (287) & $1(0.5)$ & Encephalitis \\
\hline \multicolumn{4}{|l|}{ Pembrolizumab } \\
\hline KEYNOTE-010 (REF. ${ }^{146}$ ) & 2 mg/kg, 3-weekly (339) & $3(0.9)$ & $\begin{array}{l}\text { Pneumonitis in two patients and } \\
\text { pneumonia in one patient }\end{array}$ \\
\hline KEYNOTE-010 (REF. ${ }^{146}$ ) & 10 mg/kg, 3-weekly (343) & $3(0.9)$ & $\begin{array}{l}\text { Myocardial infarction in one patient, } \\
\text { pneumonia in one patient and } \\
\text { pneumonitis in one patient }\end{array}$ \\
\hline KEYNOTE-054 (REF. ${ }^{99}$ ) & 200 mg, 3-weekly (509) & $1(0.2)$ & Myositis \\
\hline \multicolumn{4}{|l|}{ Ipilimumab plus nivolumab } \\
\hline CheckMate 067 (REF. ${ }^{168}$ ) & $\begin{array}{l}3 \mathrm{mg} / \mathrm{kg} \text { ipilimumab plus } 1 \mathrm{mg} / \mathrm{kg} \\
\text { nivolumab, 3-weekly (313) }\end{array}$ & $2(0.6)$ & $\begin{array}{l}\text { Liver failure in one patient and } \\
\text { myocarditis in one patient }\end{array}$ \\
\hline CheckMate 214 (REF.') & $\begin{array}{l}1 \mathrm{mg} / \mathrm{kg} \text { ipilimumab plus } 3 \mathrm{mg} / \mathrm{kg} \\
\text { nivolumab, 3-weekly (547) }\end{array}$ & $8(1.5)$ & $\begin{array}{l}\text { Aplastic anaemia, haemophagocytic } \\
\text { lymphohistiocytosis, lower } \\
\text { gastrointestinal haemorrhage, liver } \\
\text { failure, lung infection, pneumonia, } \\
\text { pneumonitis and unexplained sudden } \\
\text { death each in one patient }\end{array}$ \\
\hline CheckMate 227 (REF. $^{13}$ ) & $\begin{array}{l}1 \mathrm{mg} / \mathrm{kg} \text { 6-weekly ipilimumab plus } \\
3 \mathrm{mg} / \mathrm{kg} \text { 2-weekly nivolumab (576) }\end{array}$ & $7(1.2)$ & $\begin{array}{l}\text { Pneumonitis in three patients and } \\
\text { acute tubular necrosis, cardiac } \\
\text { tamponade, circulatory collapse } \\
\text { and myocarditis each in one patient }\end{array}$ \\
\hline \multicolumn{4}{|l|}{ Avelumab } \\
\hline JAVELIN solid tumour ${ }^{169}$ & 10 mg/kg, 2-weekly (184) & 0 & - \\
\hline JAVELIN Merkel 200 (REF. ${ }^{170}$ ) & 10 mg/kg, 2-weekly (88) & 0 & - \\
\hline \multicolumn{4}{|l|}{ Atezolizumab } \\
\hline $\mathrm{OAK}^{171}$ & 1,200 mg, 3-weekly (609) & 0 & - \\
\hline \multicolumn{4}{|l|}{ Durvalumab } \\
\hline ATLANTIC ${ }^{172}$ & 10 mg/kg, 2-weekly (444) & 0 & - \\
\hline
\end{tabular}

Hepatotoxicity generally has a more rapid onset than that of endocrine toxicities (within 4-9 weeks versus 7-10 weeks of commencing treatment). Other, rare toxicities (arising in $<2 \%$ of patients) include episcleritis and/or uveitis, pancreatitis, nephritis, myasthenia gravis, autoimmune autonomic ganglionopathy, Guillain-Barré syndrome (GBS) and other neuropathies, sarcoidosis-like reactions, autoimmune thrombocytopenia, toxic epidermal necrolysis and Stevens-Johnsonlike syndromes ${ }^{18}$ (TABLE 1). In a review of the literature, neurological irAEs of any grade occurred in $3.8 \%$ of patients receiving anti-CTLA- 4 antibodies; grade $\geq 3$ adverse events, which typically included headaches, encephalopathy and meningitis, occurred in $<1 \%$ of patients and had a median onset of 6 weeks after treatment initiation ${ }^{19}$.

\section{Anti-PD-1 antibodies}

In comparison with anti-CTLA- 4 antibodies, irAEs related to anti-PD-1 antibodies (such as nivolumab or pembrolizumab) are less frequent and differ in their spectrum of organ involvement (FIG. 1 b). Approximately $10 \%$ of patients receiving anti-PD- 1 antibodies have grade $\geq 3$ irAEs. Occasional any-grade toxicities (in 5-20\% of patients) include fatigue, headache, arthralgia, rash, pruritus, pneumonitis, diarrhoea and/or colitis, hepatitis and endocrinopathies (TABLE 1). Data from patients receiving anti-PD- 1 antibodies indicate that most irAEs occur within the first 6 months of treatment. Toxicities owing to anti-PD-1 or anti-PD-L1 antibodies might take longer to resolve than those related to ipilimumab, although the type of irAE is also an important determinant, and no direct comparisons of time 

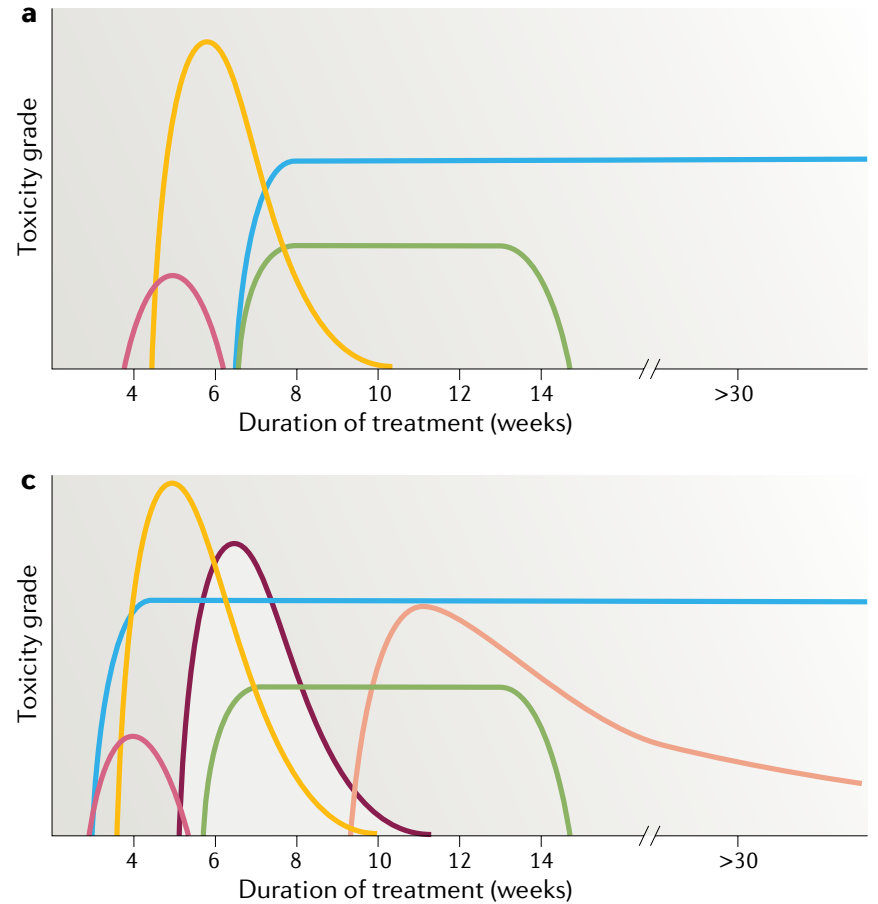

Fig. 1 | Kinetics of main irAEs. Global view of main immune-related adverse events (irAEs) in patients receiving ipilimumab (part a), antiprogrammed cell death 1 (PD-1) or anti-programmed cell death 1 ligand 1 (PD-L1) antibodies (part b) or ipilimumab plus an anti-PD-1 antibody (part c) ${ }^{152}$. Time of onset and toxicity grade are extrapolated and adapted from the references provided and are indicative only. Patients receiving anti-PD-1 or anti-PD-L1 antibodies have a lower incidence of any-grade irAEs than those receiving anti-cytotoxic T lymphocyte antigen 4 (CTLA-4) antibodies, and patients receiving a combination of both classes of agent have the highest incidence of irAEs. The incidence of grade $\geq 3$ irAEs follows a similar distribution, with $6 \%, 24 \%$ and $55 \%$ of patients receiving anti-PD-1 and/or anti-PD-L1 antibodies, anti-CTLA-4 antibodies or a combination of the two having grade $\geq 3$ irAEs, respectively ${ }^{153-155}$. Overall, irAEs in patients receiving combination immune-checkpoint inhibitors (ICls) have an earlier onset than the same irAEs in those receiving monotherapies ${ }^{1}$. Ipilimumabinduced colitis typically occurs between 4 and 8 weeks after first infusion and is almost never seen $>2$ months after the last treatment dose ${ }^{156}$ (median reported time between colitis and last dose of ipilimumab is 11 days (range $0-59))^{157,158}$. ICl-induced hypophysitis typically emerges between 6 and 14 weeks of treatment, with a median time of onset of 8 weeks in a large cohort of patients with metastatic melanoma receiving ipilimumab ${ }^{159,160}$. The median reported time to new onset or exacerbation of pre-existing abnormalities in thyroid function becoming detectable on biochemical tests is typically 4-7 weeks after treatment onset in patients receiving $\mathrm{ICls}^{161}$. ICl-induced hepatitis seems to have a more variable onset time, with

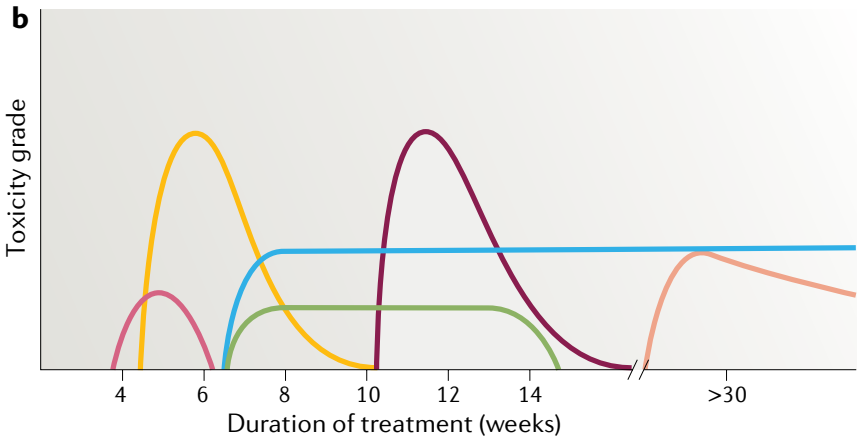

\begin{tabular}{|ll|}
\hline Colitis & Endocrinopathy \\
- Liver toxicity & Skin, rash \\
or pruritus & Nephritis \\
\hline
\end{tabular}

data from small series of patients indicating that abnormal liver test results can emerge between 1 and 14 weeks of treatment, with median onset times ranging from 3 to 9 weeks ${ }^{162,163}$. Neurological irAEs are reported to occur between 1 and 7 weeks of treatment initiation in patients receiving ipilimumab, albeit with a slightly earlier onset ( 2 to 6 weeks) for myasthenia gravis (not shown) $65,4.5$ weeks for anti-PD-1 antibodies and 2 weeks in patients receiving combination $\mathrm{ICl}^{19}$. Data from a systematic review confirmed this interval, indicating a median onset of neurological irAEs of 6 weeks after treatment, with partial or complete recovery of adequately managed irAEs expected within 4 weeks ${ }^{91}$. A 2-12-week interval between treatment onset and the development of acute interstitial nephritis (AIN) was reported in patients receiving ipilimumab, with delayed AIN occurring up to 26 weeks after treatment onset ${ }^{95}$. A longer delay of AIN onset, from 3 to 12 months, was reported in patients receiving anti-PD-1 and/or antiPD-L1 antibodies ${ }^{164}$. Rheumatic irAEs are a new nosological entity with a median time to onset of 7.3 weeks, with, once again, a wide dispersion of onset times, with events described $>50$ weeks after ICl initiation ${ }^{165}$. Pneumonitis is exceptionally rare in patients receiving anti-CTLA-4 antibodies as opposed to those receiving anti-PD-1 or anti-PD-L1 antibodies or ICl combination therapy. A median time to onset of 2.5 months has been reported for pneumonitis among patients receiving any $\mathrm{ICls}$ (ranging from 2 to 24 months), with expected resolution of symptoms of mild to moderate pneumonitis within 2-8 weeks ${ }^{62}$. Another study revealed a shorter time to onset of pneumonitis, starting as early as 9 days from initial infusion of an anti-PD-1 antibody ${ }^{32}$. to resolution of irAEs caused by different agents are currently available ${ }^{20}$.

In the milestone CheckMate 066 trial, comparing the efficacy and safety of nivolumab with that of dacarbazine, three-quarters of patients with previously untreated $B R A F$-wild-type, advanced-stage melanoma had an adverse event of any grade, with a similar incidence of grade $\geq 3$ toxicities of $11.7 \%$ and $17.6 \%$ with nivolumab and dacarbazine, respectively ${ }^{21}$. The most common irAEs among patients in the nivolumab group were endocrinopathies (mostly thyroiditis), pneumonitis, hepatitis, diarrhoea and colitis. Fatigue, pruritus and nausea (of any grade) were also reported in $>15 \%$ of patients ${ }^{21}$.
Pembrolizumab seems to have a somewhat similar toxicity profile to that of nivolumab, as demonstrated by the safety profiles of cohorts receiving this agent in the various prospective KEYNOTE trials with data published so far (TABLE 1). In the phase III KEYNOTE-407 trial $^{22}$, the efficacy of pembrolizumab plus carboplatin and paclitaxel or nab-paclitaxel versus that of carboplatin and paclitaxel or nab-paclitaxel alone was investigated in patients with previously untreated metastatic squamous NSCLC. This trial did not reveal any increase in grade $\geq 3$ adverse events (which occurred in $69.8 \%$ of patients receiving pembrolizumab plus chemotherapy versus $68.2 \%$ of those receiving chemotherapy alone). 
Pneumonitis and autoimmune hepatitis were slightly more frequent among patients in the pembrolizumab plus chemotherapy group than in the chemotherapy alone group (2.5\% versus $1.1 \%$ and $1.8 \%$ versus $0 \%$, respectively $)^{22}$. Discontinuation of treatment owing to adverse events was more frequent in the pembrolizumab plus chemotherapy group than in the chemotherapy group ( $13.3 \%$ versus $6.4 \%)$; however, patients in the pembrolizumab plus chemotherapy groups still had improved overall survival (OS) outcomes ${ }^{22}$. Importantly, $>97 \%$ of patients in both groups had toxicities, with more than two-thirds having at least one grade $\geq 3$ adverse event, albeit with considerable differences in the types of events ${ }^{22}$.

\section{Ipilimumab plus nivolumab}

The combination of an anti-CTLA- 4 antibody and an anti-PD-1 antibody increases both the incidence and severity of irAEs. For example, a phase II trial comparing the efficacy of ipilimumab plus nivolumab with that of ipilimumab alone in patients with $B R A F$-wildtype melanoma revealed a much greater incidence of grade $\geq 3$ toxicities in the combination group (54\% versus $24 \%)^{23}$. IrAEs also tended to occur earlier in the course of treatment with ipilimumab plus nivolumab compared with ipilimumab monotherapy (FIG. 1c). The most frequently occurring irAE was colitis (in $17 \%$ of patients), followed by dermatological, endocrine and hepatic events ${ }^{23}$. In the CheckMate 067 trial $^{7}$, grade $\geq 3$ treatment-related adverse events occurred in $55 \%$ of patients in the group receiving ipilimumab $(3 \mathrm{mg} / \mathrm{kg})$ plus nivolumab (1 mg/kg), compared with $27.3 \%$ and $16.3 \%$ in the ipilimumab and nivolumab monotherapy groups, respectively. The CheckMate 214 trial $^{9}$, in which the efficacy of ipilimumab $(1 \mathrm{mg} / \mathrm{kg})$ plus nivolumab $(3 \mathrm{mg} / \mathrm{kg})$ was compared with that of the TKI sunitinib in patients with advanced-stage RCC, was the first to show an OS advantage in this disease. The respective frequencies of the most common toxicities (colitis and/or diarrhoea, dermatological, endocrine and hepatic events) were lower than those seen in patients with advanced-stage melanoma treated with the same combination ${ }^{8}$. More patients discontinued the combination treatment owing to adverse events ( $22 \%$ versus $12 \%$ ), although fewer patients receiving ipilimumab plus nivolumab had treatment-related grade $\geq 3$ adverse events, relative to those in the sunitinib group ( $46 \%$ versus $63 \%$ ). This finding is mostly explained by the reduced dose of ipilimumab used in CheckMate 214 relative to CheckMate 067, together with the narrower therapeutic range of sunitinib. Patient demographics in these trials ${ }^{7,8}$ were similar in both groups and, thus, the different toxicity profiles are unlikely to be the result of disparities in patient characteristics. The potential contribution of tumour-induced immunomodulation to differences in the severity of adverse events between patients with RCC and those with melanoma is unknown. Once again, premature discontinuation of treatment owing to irAEs did not have a negative effect on patient outcomes, similar to the experience with patients with melanoma. A phase III trial comparing the two ipilimumab plus nivolumab regimens is currently being conducted in patients with metastatic melanoma (NCT02714218), in whom $3 \mathrm{mg} / \mathrm{kg}$ ipilimumab plus $1 \mathrm{mg} / \mathrm{kg}$ nivolumab is still the standard-of-care approach. Similar incidences of irAEs have been reported in a systematic review of data from patients receiving ICIs for the treatment of metastatic RCC or melanoma ${ }^{24}$; however, the only data on combinations of ICIs in patients with RCC to be included in this analysis came from the CheckMate 016 phase I trial ${ }^{25}$.

In the phase I CheckMate 012 trial involving patients with advanced-stage NSCLC ${ }^{12}, 6$-weekly versus 12 -weekly dosing intervals of $1 \mathrm{mg} / \mathrm{kg}$ ipilimumab were investigated in patients also receiving 2 -weekly $3 \mathrm{mg} / \mathrm{kg}$ nivolumab; similar numbers of patients (14 (37\%) versus $13(33 \%))$ had grade $\geq 3$ adverse events. In the phase III CheckMate 227 trial $^{13}$, patients with NSCLCs harbouring a high tumour mutational burden (TMB; $\geq 10$ mutations per $\mathrm{Mb}$ ) seemed to be more sensitive to the $1 \mathrm{mg} / \mathrm{kg}$ ipilimumab plus $3 \mathrm{mg} / \mathrm{kg}$ nivolumab combination regimen (1-year progressionfree survival $42.6 \%$ in patients with a high TMB versus $30.9 \%$ in all patients), although according to a subgroup analysis this improved efficacy came at the cost of a higher risk of grade $\geq 3$ adverse events ( $37 \%$ in the high TMB subgroup versus $18 \%$ among all patients receiving this combination $)^{13}$. Treatment-related mortality was slightly greater than $1 \%$ in both the immunotherapy and conventional chemotherapy arms ${ }^{13}$. As expected, the causes of treatment-related deaths were different in each arm, with vascular and/or thrombotic, infectious and haematological (agranulocytosis and thrombocytopenia) adverse events as the leading causes in the conventional chemotherapy group and severe irAEs (such as myocarditis, pneumonitis and renal insufficiency) in the combination immunotherapy group (TABLE 1).

\section{Anti-PD-L1 antibodies}

In a systematic review, Khoja et al. ${ }^{26}$ were unable to discriminate the adverse event profiles of the anti-PD-L1 antibodies (BMS-936559 and atezolizumab) from those of anti-PD-1 antibodies. However, the authors advanced the hypothesis that anti-PD-L1 antibodies, theoretically, might be less toxic owing to the preservation of PD-L2 signalling, thus better preserving immune homeostasis. A reliable comparison of the risk of adverse events is difficult owing to the different indications and tumour types, outside of NSCLC, for which anti-PD-1 antibodies and anti-PD-L1 antibodies are FDA approved ${ }^{27}$. Nonetheless, a meta-analysis by Khunger et al. ${ }^{28}$ that included data from 19 clinical trials focusing on anti-PD-1 and/or anti-PD-L1 antibody-associated lung toxicities revealed a significantly higher incidence of pneumonitis among patients receiving anti-PD- 1 antibodies $(4.9 \%$ versus $1.9 \%$; $P<0.001)$. The severity of pneumonitis was also higher in this group than in cohorts receiving anti-PD-L1 antibodies. An increased incidence of pneumonitis was also reported in a meta-analysis of data from $>5,000$ patients with NSCLC who received either anti-PD-1 or anti-PD-L1 antibodies (4\% versus $2 \% ; P=0.01)^{29}$. 
As strongly suggested by a meta-analysis described in the next section ${ }^{30}$, this slightly higher incidence of toxicities associated with anti-PD-1 antibodies might reflect the adverse effects of pembrolizumab rather than nivolumab, with the former having a higher adverse event rate, and the better safety profile of atezolizumab among PD-L1 inhibitors in this comparison.

A subset of patients receiving the anti-PD-L1 antibody avelumab have infusion-related reactions (IRRs). These IRRs are predominantly grade $1-3$ in severity, manifest as chills, pyrexia and flushing and most likely reflect the activation of innate immunity owing to the presence of an intact, fully human Fc region. These adverse events affect approximately one-quarter of all patients and generally occur during or just after the first four infusions of the drug ${ }^{31}$. Reducing the rate of infusion, temporarily suspending the infusion, administering premedication consisting of paracetamol and antihistamines or, if required, administering low-dose steroids are all effective methods of managing this type of adverse event ${ }^{32}$.

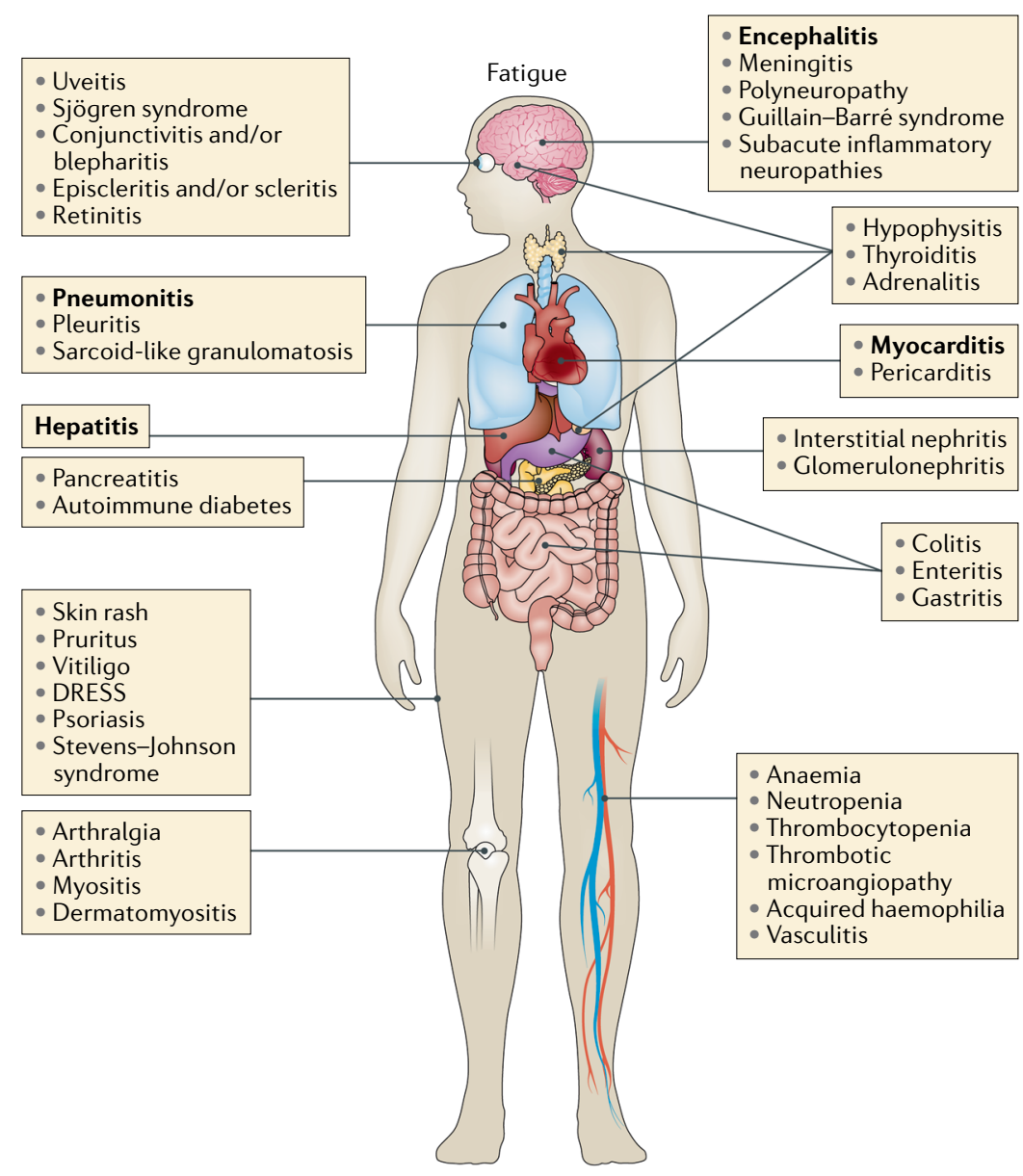

Fig. 2 | The spectrum of irAEs by affected organ or organs. Immune-checkpoint inhibitors (ICls) promote the activation and expansion of T cells. Owing to the diversity of the T cell population and the ability of these cells to infiltrate most organs, ICls can cause a wide range of immune-related adverse events (irAEs), and these can affect virtually any organ. The most frequently affected organs and the most common specific irAEs are highlighted in boxes. irAEs contributing to most fatalities are highlighted in bold. DRESS, drug rash with eosinophilia and systemic symptoms.

\section{Comparative safety}

In a systematic review and meta-analysis of data from 36 comparative phase II and III randomized trials ( $n=15,370)$, investigators compared the safety profiles of several ICIs ${ }^{30}$. Atezolizumab had the best overall safety profile, as indicated by the lowest risk of adverse events of any grade and grade $\geq 3$ adverse events $(66.4 \%$ and $15.1 \%$, respectively), followed by nivolumab (71.8\% and $14.1 \%)$, pembrolizumab (75.1\% and $19.8 \%$ ) and ipilimumab (86.8\% and $28.6 \%)$. Treatment with atezolizumab conferred the highest risk of hypothyroidism, and nausea and vomiting. The predominant treatment-related adverse events associated with pembrolizumab were arthralgia, pneumonitis and hepatic toxicities, while nivolumab mainly caused endocrine toxicities. The main treatment-related adverse events associated with ipilimumab were skin, gastrointestinal and renal toxicities $^{30}$. Treatment with nivolumab resulted in the lowest risk of adverse events among all ICIs in the subgroup of patients with NSCLC. So far, different risk factors for anti-PD-1-related pneumonitis have been reported in different published retrospective analyses. A retrospective analysis of data from 123 patients with NSCLC demonstrated that pre-existing pulmonary fibrosis (even at a mild stage) substantially increases the risk of antiPD-1-related pneumonitis in patients with NSCLC ${ }^{33}$. Indeed, pneumonitis was observed in half of the patients with a fibrosis score of 1 and in all patients with a fibrosis score of 2 or 3 (indicating a greater amount of scarring $)^{33}$. The findings of another series of 915 patients showed that worsening cases of anti-PD-1-induced and/or anti-PD-L1-induced pneumonitis were more frequent in patients who are either current or former smokers, as well as in those with underlying lung comorbidities $^{32}$.

Nevertheless, pneumonitis has been suggested to be predictive of favourable outcomes in patients receiving anti-PD-1 antibodies $^{34}$. Moreover, an association between nivolumab-related irAEs and efficacy has been reported in patients with NSCLC ${ }^{35}$. Most trials exclude patients with interstitial lung disease owing to an increased risk of adverse events. For this reason, we propose that, in patients with a higher risk of antiPD-1 antibody-induced and/or anti-PD-L1 antibodyinduced pneumonitis (owing to pre-existing pulmonary fibrosis and/or other lung comorbidities), ICIs should not be entirely avoided but rather that the ICI with the lowest risk of lung toxicities (for example, nivolumab or atezolizumab over pembrolizumab) should be selected if possible and especially if used as a second-line treatment.

The same analogy could be applied to selecting the most appropriate ICI according to the pre-existing risk factor profiles of each patient. Following a thorough clinical assessment, the ICI associated with the lowest possible risk of the most likely adverse events could be selected for each patient.

\section{Types of irAE}

The spectrum of organ systems affected by irAEs is very broad; as such, toxicities can affect almost any organ, with varying frequencies and severities (FIG. 2). Clinicians should be aware that rare, yet life-threatening irAEs are 
constantly being reported. This ever-changing body of evidence creates a challenge to the successful diagnosis and management of patients with these events.

\section{Cutaneous irAEs}

Cutaneous irAEs affect between one-third and more than half of all patients receiving ICIs ${ }^{36}$. Rash, pruritus and vitiligo are the most widely reported skin toxicities and are similar in patients receiving anti-CTLA-4 antibodies and those receiving anti-PD-1 antibodies ${ }^{37}$ (TABLE 1). The occurrence of vitiligo has been linked to better tumour responses and outcomes in patients with advanced-stage melanoma ${ }^{38}$. In a retrospective study ${ }^{39}$, the emergence of lichenoid and spongiotic histopathological patterns of dermatitis was associated with favourable outcomes in a small cohort of patients receiving antiPD-1 or anti-PD-L1 antibodies for the treatment of various malignancies. The majority of ICI-induced cutaneous irAEs are maculopapular and affect $<30 \%$ of the body surface area (BSA), with follicular, pustular, vesicular and acneiform presentations all being reported ${ }^{40}$.

With effective management, ICI-induced rash can almost fully resolve within 1-2 months, although some patients have persistent and/or recurrent low-grade cutaneous toxicities after completing subsequent courses of treatment ${ }^{41}$. However, extensive (covering $>30 \%$ of BSA), exfoliative, ulcerative or bullous ICI-induced dermatitis is not uncommon: grade $\geq 3$ cutaneous irAEs are typically observed in $2-3 \%$ of patients receiving ICI monotherapies and $4-10 \%$ receiving combinations of ICIs ${ }^{42}$. A pooled analysis of mucocutaneous irAEs revealed other rare, less-severe toxicities including xerosis, stomatitis, urticaria, photosensitivity reactions, changes in hair colour, alopecia areata and hyperhidrosis ${ }^{37}$. Other cutaneous presentations include ICI-induced dermatomyositis, drug response with eosinophilia and granulomatous, lichenoid, panniculitis-like and lupus-like reactions ${ }^{40}$.

Particular caution must be taken in the management of patients with either active or past psoriasis or even in those with a family history of psoriasis; exacerbation of previous psoriasis has been described in five patients receiving pembrolizumab, nivolumab or durvalumab ${ }^{43}$. Patients receiving anti-PD-1 or anti-PD-L1 antibodies seem to have a broader range of skin toxicities than those receiving anti-CTLA- 4 antibodies as monotherapies; however, a possible bias exists in the reporting of these events owing to the wider use of anti-PD-1 and anti-PD-L1 antibodies in clinical practice. Skin reactions caused by antibodies targeting PD-1 or PD-L1 also tend to have a delayed onset in comparison with those caused by anti-CTLA- 4 monotherapies either during prolonged treatment or after treatment has been discontinued $^{42,44,45}$. Treatment with ICI combination therapy is associated with earlier-onset skin toxicities with an increased incidence and greater severity than those associated with monotherapies ${ }^{46}$.

\section{Lower digestive tract irAEs}

As previously mentioned, colitis is the most frequently observed irAE in patients receiving ipilimumab, occurring in $10-20 \%$ of patients ${ }^{47}$ (TABLE 1). Endoscopic examinations usually reveal a mucosa with diffuse ulceration and oedema that can possibly affect the entire colon rather than a segment. Associated diffuse enteritis is also present in one-quarter of patients and can occur in the absence of colitis ${ }^{48}$. Enteritis should be considered in patients with diarrhoea with unexplained weight loss and an endoscopic examination showing a colonic mucosa with no apparent pathological alterations.

Examinations of colon biopsy samples from patients with ICI-induced colitis typically reveal a mixed lymphocytic and neutrophilic infiltrate with apoptotic mucosal epithelial cells and crypt abscesses. The presence of plasma cells and eosinophils is also common. Owing to the acute nature of this irAE, however, the structure of the epithelium is usually preserved, in contrast to the pathology of inflammatory bowel disease (IBD). Even in the absence of macroscopic disease, biopsy samples should be obtained in order to assess the possibility of underlying microscopic colitis ${ }^{49}$. Histological features of active inflammation encompassing extensive mucosal distortion with crypt abscesses and plasmacytosis have been correlated with a higher risk of recurrence. The presence of large ( $>1 \mathrm{~cm}$ in diameter) and deep $(>2 \mathrm{~mm})$ ulcerations, together with extensive mucosal involvement, is predictive of the requirement for biological therapies according to data from a large retrospective study $^{50}$. In another retrospective analysis ${ }^{51}$, adding infliximab to corticosteroids as an initial treatment was shown to be associated with a shorter time to symptom resolution and a reduced need for steroids in patients with ICI-induced high-grade colitis. Although prospective data are still awaited, evidence suggests the beginning of a paradigm shift towards more selective management of high-grade ICI-induced colitis using biological agents as first-line therapies. This strategy has the potential to prevent adverse outcomes by avoiding the development of a chronic inflammatory state at an early stage.

CTLA-4 inhibition can result in a loss of selftolerance of mucosal flora and autoantigens. Depletion of $\mathrm{T}_{\text {reg }}$ cells in the bowel mucosa seems to have an important role in this process ${ }^{52}$. This phenomenon can also be influenced by genetic predisposition; for example, the presence of a CTLA4 polymorphism (Y60C) has been shown to increase the risk of early onset Crohn's disease ${ }^{53}$.

Qualitative alterations in the gut microbiota of mice loaded with certain strains of bacteria, such as Bacteroides fragilis and Burkholderia cepacia, have been shown to protect against the development of antiCTLA-4 antibody-induced colitis and are associated with an enhanced antitumour effect ${ }^{54-56}$. In humans, a prospective study of the composition of the colonic microbiota at the start of treatment demonstrated that over-representation of bacteria of the genus Bacteroides was associated with a lower incidence of colitis. In addition, progression to colitis can successfully be predicted by analysing the composition of the microbiota: a paucity of several bacterial pathways, such as polyamine transport and vitamin B biosynthesis, is correlated with an increased risk of colitis ${ }^{57}$. Furthermore, two patients with ICI-induced colitis that was refractory to steroids, anti-TNF antibodies and anti-integrin antibodies have been successfully treated with transplantation of faecal 
microbiota from healthy unrelated donors ${ }^{58}$. Follow-up colon biopsy samples from these two patients had altered immune infiltrates, with a substantial decrease in infiltrating $\mathrm{CD}^{+} \mathrm{T}$ cells in both patients and an increase in infiltrating $\mathrm{T}_{\text {reg }}$ cells in one $\mathrm{e}^{58}$. In a phase I trial ${ }^{59}$, a positive correlation was reported between high serum IL-17 levels before treatment with ipilimumab and the development of colitis. Whether or not serum IL-17 titres can be used as a reliable predictor of ipilimumab-induced colitis remains unknown.

The underlying biology of anti-PD-1 antibodyinduced colitis is different to that of ipilimumab-induced colitis. PD-1 has been shown to be upregulated in gut biopsy samples from patients with IBD, and anti-PD-1 antibodies have been shown to lead to severe enteritis in ovalbumin-specific transgenic mouse models ${ }^{60,61}$. Nevertheless, anti-PD-1 antibody-induced colitis and/or enteritis is much less prevalent than ipilimumab-induced colitis, thus highlighting a putative redundant role of this pathway in the maintenance of gut immune homeostasis.

\section{Pulmonary irAEs}

Patients receiving anti-PD-1 antibodies are more likely to have any-grade immune-related pneumonitis than those receiving anti-CTLA- 4 antibodies $(1-5 \% \text { versus }<1 \%)^{62}$ (TABLE 1). This life-threatening complication is often challenging to diagnose, especially among patients with lung cancer who also have pre-existing chronic lung disease. Several patterns of radiological presentation have been reported, including cryptogenic organizing pneumonia (COP) as the most frequent but also nonspecific interstitial pneumonia, hypersensitivity pneumonitis, acute interstitial pneumonia, sarcoid-type reactions and acute respiratory distress syndrome ${ }^{62}$. COP-like radiological presentations are more common in patients with NSCLC and are a potential predictor of the need for immunosuppression early in the course of treatment ${ }^{32}$. In a retrospective study of data from 43 patients with anti-PD-1 or anti-PD-L1 antibody-induced pneumonitis, 12 (27\%) had grade $\geq 3$ pneumonitis, 5 (12\%) of whom ultimately had a fatal outcome during treatment of this adverse event ${ }^{32}$. One death was attributed to cancer progression, although three of the other patients died from infectious complications related to drug-induced immunosuppression and a fourth from refractory pneumonitis. Importantly, 7 (64\%) of the 12 patients with grade $\geq 3$ pneumonitis had improvements upon treatment with steroids and withdrawal of ICIs, while the patients who died did so despite additional immunosuppression with infliximab and/or cyclophosphamide either owing to refractory disease or infectious complications. These findings highlight the tremendous need to determine the optimal management of frail patients requiring immunosuppression and to develop predictive tools to guide the optimal use of agents such as steroids and/or infliximab ${ }^{32}$.

The demographics of patients with pneumonitis have provided important insights into the pathogenesis of this irAE. Anti-PD-1 or anti-PD-L1 antibody-induced pneumonitis is more frequent in the first-line setting and has both a greater incidence and severity in patients with NSCLC than in those with melanoma ${ }^{28,63}$. These observations suggest that chemotherapy-induced lung inflammation, previous radiotherapy, pre-existing lung disease and smoking are all risk factors that contribute to the occurrence, severity and prognosis of this irAE. A decreased prevalence of ICI-induced pneumonitis in the second-line setting could be explained by more patients in this setting having suppressed or compromised immune system function owing to the typically increased tumour burdens and prior treatment with chemotherapy. Few reports describing the histopathology of patients with ICI-induced pneumonitis are currently available. The autopsy of a 35-year-old patient who died with nivolumab-associated pneumonitis (after melanoma progression on first-line dacarbazine followed by second-line ipilimumab) revealed diffuse alveolar damage, interstitial CD8 ${ }^{+} \mathrm{T}$ cell-enriched lymphocytic infiltrates and panlobular sarcoid-like granulomatous lesions owing to pneumonitis ${ }^{64}$. Bronchoalveolar lavage of patients with anti-PD-1 or anti-PD-L1 antibodyinduced pneumonitis can reveal lymphocytosis with variable $\mathrm{CD} 4^{+} \mathrm{T}$ cell:CD8 ${ }^{+} \mathrm{T}$ cell ratios; inverted $\mathrm{CD} 4^{+}$ $\mathrm{T}$ cell:CD8 ${ }^{+} \mathrm{T}$ cell ratios have been reported in patients with sarcoid-like presentations ${ }^{65,66}$.

\section{Hypophysitis}

Hypophysitis, a condition involving inflammation of the pituitary gland, is rare in patients receiving anti-PD-1 antibodies but much more common in those receiving ipilimumab, with an incidence of $12.0-13.3 \%$ in the real-world setting ${ }^{67,68}$. The development or worsening of fatigue, weakness, headache, visual disturbances, arterial hypotension and nausea in patients receiving ICIs should raise the suspicion of hypophysitis and requires immediate assessment of pituitary functional status. Pituitary MRI should be performed early in the course of the condition in order to eliminate pituitary metastases as a differential diagnosis and to assess the degree of pituitary enlargement owing to potential mass effects with compression of the optic chiasm. Enlargement of the pituitary stalk resolves within 6 weeks in the majority of patients ${ }^{68,69}$. Adrenal insufficiency of central (pituitary) origin is usually persistent, even after pituitary inflammation has regressed ${ }^{70}$. With proper management, however, approximately one-third to half of all patients recover function of the pituitary-thyroid axis, and the gonadal axis is restored in half of all men with this irAE. Interestingly, diabetes insipidus is an uncommon comorbidity in this setting compared with other autoimmune diseases ${ }^{68}$. Iwama et al..$^{71}$ published interesting findings on the pathogenesis of ipilimumab-induced hypophysitis. In a case series of seven patients, all patients developed circulating autoantibodies to thyroid-stimulating hormone (TSH)-secreting cells and, less frequently, to follicle-stimulating hormone (FSH)-secreting or adrenocorticotropic hormone-secreting cells ${ }^{71}$, all of which are associated with defects in their respective functional axes. These investigators also found that human prolactin-secreting and TSH-secreting pituitary cells express CTLA-4 $\left(\right.$ REF. $\left.^{71}\right)$. Anti-CTLA- 4 antibody-induced hypophysitis might be caused by direct binding of the monoclonal antibody to the CTLA-4 antigens present in the pituitary gland rather than a consequence of $\mathrm{T}$ cell infiltration following activation of immune cells 
within the TME or the lymphatic system (implying a loss of self-tolerance). In support of this hypothesis, Caturegli et al. ${ }^{72}$ confirmed the expression of CTLA-4 in nonmalignant pituitary cells as well as in pituitary adenomas in an autopsy series of patients who received anti-CTLA-4 antibodies. Ipilimumab is an immunoglobulin G1 (IgG1) antibody that is, therefore, capable of activating the classical complement cascade; thus, a type II hypersensitivity reaction could have a role in the pathogenesis of hypophysitis (via antibody-dependent complement-mediated cytotoxicity (CDC)). In support of this hypothesis, a lower frequency of tremelimumabassociated hypophysitis was observed in clinical trials involving this agent ${ }^{73}$, which is an IgG2 anti-CTLA-4 antibody and is thus less likely to activate the complement cascade. However, the high density of CTLA-4 expression in the pituitary gland might nonetheless lead to hypophysitis, even with the use of tremelimumab, by increasing the risk of an antibody-dependent cellular cytotoxicity (ADCC). This suggestion is supported by the presence of a higher level of pituitary CTLA-4 expression in a patient with clinical and histological evidence of severe hypophysitis associated with tremelimumab in the aforementioned autopsy series ${ }^{72}$. Rare incidences of anti-PD-1 antibody-induced hypophysitis have also been reported ${ }^{74}$. Overall, ICI-induced hypophysitis seems to occur owing to a combination of ADCC, CDC and direct cell-mediated cytotoxicity owing to a loss of self-tolerance.

\section{Thyroid irAEs}

In contrast to hypophysitis, thyroid dysfunction seems to be more frequently associated with antibodies targeting PD-1, as opposed to CTLA-4 (REF. ${ }^{75}$ ). Interestingly, to date, thyroid dysfunctions are the only endocrine irAEs reported to be associated with anti-PD-L1 antibodies $^{75}$. In the real-world setting, nearly $20 \%$ of patients receiving anti-PD-1 antibodies present with thyroid dysfunction, which typically occurs early in the course of treatment, with a median onset of 6 weeks following the first infusion ${ }^{76,77}$. Most thyroid irAEs are asymptomatic, presenting with mild thyrotoxicosis or primary hypothyroidism related to destructive thyroiditis or, less commonly, thyrotoxicosis related to autoimmune thyroid disease (Graves disease) ${ }^{77}$. In a published study in which thyroid function was monitored prospectively in patients with melanoma receiving pembrolizumab ${ }^{76}$, most patients presenting with hyperthyroidism subsequently developed hypothyroidism within 1-3 months. ICIs can be continued in the majority of patients who develop hyperthyroidism, and $\beta$-blockers can be prescribed for symptom relief. In those with persistent hypothyroidism, levothyroxine substitution should be initiated after ruling out the possibility of adrenal insufficiency. Data from studies of the pathophysiology of ICI-induced thyroid dysfunction are currently lacking; future cytological and molecular analyses of thyroid fine-needle aspirates could address this issue. The prognosis of patients who recover from thyroid dysfunction is uncertain, with some authors reporting full recovery (true thyroiditis $)^{78}$ and others persistent hypothyroidism (Hashimoto-like course) in small series of patients ${ }^{77}$.

\section{Hepatic irAEs}

Hepatic irAEs seem to have an almost equal incidence in patients receiving ipilimumab and in those receiving antiPD- 1 antibodies as monotherapies (occurring in 5-10\% of patients), albeit with a tendency towards greater severity in patients receiving ipilimumab ${ }^{79}$ (TABLE 1). Patients with ICI-induced hepatitis most commonly present with isolated elevations of liver transaminases, and these symptoms are often limited after treatment discontinuation $^{80}$. Nevertheless, in daily clinical practice, growing evidence of severe cases associated with liver dysfunction (hyperbilirubinaemia and coagulopathy) is emerging, as is evidence of rare life-threatening forms associated with acute liver failure ${ }^{81}$. According to current guidelines, ICIs should be suspended in patients with grade 2 elevations in liver transaminase levels (defined as 2-5 times the upper limit of normal) and permanently stopped when transaminase levels exceed this cut-off ${ }^{80,81}$. Liver biopsy samples should be obtained from all patients with moderate to severe elevations of liver transaminase levels (greater than three times the upper limit of normal) to rule out an alternative cause.

In a phase I trial involving patients with $\mathrm{RCC}^{25}$, the combination of $3 \mathrm{mg} / \mathrm{kg}$ ipilimumab plus $1 \mathrm{mg} / \mathrm{kg}$ nivolumab was more toxic than the reverse $(1 \mathrm{mg} / \mathrm{kg}$ and $3 \mathrm{mg} / \mathrm{kg}$ doses of ipilimumab and nivolumab, respectively) mainly owing to hepatotoxicities, with similar levels of efficacy. Almost 1 in 5 patients in the $3 \mathrm{mg} / \mathrm{kg}$ ipilimumab plus $1 \mathrm{mg} / \mathrm{kg}$ nivolumab group had grade 3-4 hepatitis (17.0\% versus $6.4 \%$ of patients receiving $1 \mathrm{mg} / \mathrm{kg}$ ipilimumab plus $3 \mathrm{mg} / \mathrm{kg}$ nivolumab), among other toxicities $^{25}$. Thus, the $1 \mathrm{mg} / \mathrm{kg}$ ipilimumab plus $3 \mathrm{mg} / \mathrm{kg}$ nivolumab combination was chosen for comparison with sunitinib in the phase III CheckMate 214 trial.

Typical histological features of hepatic irAEs include a mixed panlobular immune cell infiltrate with lymphocytic predominance with, in some patients, signs of focal to confluent necrosis ${ }^{82}$. Cholestasis consistent with a portal mononuclear infiltrate can be observed in patients with severe hepatic irAEs following treatment with ipilimumab $^{83}$. Another interesting finding is the presence of fibrin-ring histiocytes surrounding lipid vacuoles in two patients with hepatitis related to ipilimumab plus nivolumab combination therapy ${ }^{84}$.

\section{Cardiac irAEs}

In a retrospective registry study of data from eight clinical centres $^{85}$, investigators estimated the prevalence of ICIinduced myocarditis to be $1.14 \%$. ICI-induced myocarditis is widely feared owing to the high risk of death associated with this irAE. An interrogation of the pharmacovigilance databases of Bristol-Myers Squibb (the manufacturer of nivolumab and ipilimumab) revealed 18 instances of severe ICI-related myocarditis among 20,594 patients $(0.09 \%)^{86}$. The incidence of severe myocarditis was higher in patients receiving a combination of nivolumab and ipilimumab $(0.27 \%)$ than in those receiving nivolumab alone $(0.06 \%)^{86}$. Similarly, myocarditis seemed more likely to be fatal in patients receiving combination ICI therapy ( 5 fatalities versus 1 fatality or mortality of $0.27 \%$ versus $0.06 \% ; P<0.001)^{86}$. In a review of the WHO database published in 2018 , mortality of $46 \%$ was reported in a total 
of 101 patients with ICI-induced myocarditis (67\% with combination ICI therapy versus $36 \%$ with anti-PD- 1 or anti-PD-L1 antibodies as monotherapy) ${ }^{87}$.

The clinical presentation of ICI-induced myocarditis is heterogeneous, ranging from chest pain to acute dyspnoea and/or acute circulatory collapse ${ }^{88}$. Fatal cases of myocarditis have been reported after a single dose of $3 \mathrm{mg} / \mathrm{kg}$ ipilimumab plus $1 \mathrm{mg} / \mathrm{kg}$ nivolumab, and emerging data suggest that myocarditis usually occurs early in the course of treatment: $81 \%$ of events occur within the first 4 cycles (at a median of 34 days from treatment initiation) ${ }^{85,86,89}$.

Mechanisms of ICI-induced myocarditis again implicate a loss of self-tolerance. Histological autopsy examination of a patient who died from anti-PD-1-induced myocarditis showed a predominantly $\mathrm{CD}^{+} \mathrm{T}$ cell infiltrate, together with some $\mathrm{CD} 4^{+} \mathrm{T}$ cells and sparsely distributed $\mathrm{B}$ cells ${ }^{90}$. In another report, observations from two patients confirmed the existence of shared $\mathrm{T}$ cell receptor clonality between tumour-infiltrating and myocardial-infiltrating T cells without signs of IgG deposits in the heart ${ }^{86}$.

Any signs of cardiac insufficiency or chest discomfort in a patient receiving ICIs should prompt a full cardiac assessment. In addition, a baseline electrocardiogram is advised before starting treatment with ICIs because cardiac toxicities could manifest as isolated arrhythmias. Furthermore, the risk of silent myocardial injury during the course of treatment justifies regular monitoring of serum troponin levels.

\section{Neurological irAEs}

Neurological irAEs can affect the central or the peripheral nervous system, encompassing a wide range of clinical presentations ${ }^{19,91}$. Kao et al..$^{92}$ published a retrospective cohort study describing the development of neurological complications with an incidence of $2.9 \%$ (10/347) in patients receiving anti-PD-1 antibodies. Half of the patients presented with concomitant irAEs affecting other organs, such as hypothyroidism, colitis and hepatitis ${ }^{92}$. Collectively, the neurological and neuromuscular complications of ICI treatment include myopathies, neuromuscular junction disorders, peripheral neuropathies (PNPs; including axonal and demyelinating polyradiculoneuropathies), length-dependent and non-length-dependent neuropathies, asymmetric mononeuritis multiplex, cerebellar ataxia, retinopathy, bilateral internuclear ophthalmoplegia and headache ${ }^{93}$. We emphasize, however, that a growing body of literature on neurological irAEs with pleomorphic presentations continues to accumulate ${ }^{94}$.

Encephalitis and/or aseptic meningitis. Central nervous system (CNS) symptoms, such as seizures, confusion, ataxia or even amnesia, should prompt an extensive diagnostic work-up aimed at excluding the possibility of not only infectious, metabolic and/or toxic complications but also paraneoplastic phenomena or CNS metastasis. For example, a diagnosis of autoimmune encephalitis as an adverse effect of ICIs is made by exclusion. Examinations of the cerebrospinal fluid (CSF) might reveal polyclonal lymphocytosis with an elevated protein concentration despite no evident abnormalities on brain MRI in some patients. Otherwise, brain MRI reveals hyperintense T2 signals in affected regions, and an observation of meningeal thickening and/or enhancement might redirect the diagnosis towards aseptic meningitis or meningoencephalitis depending on the clinical presentation. An autoantibody panel should be used to analyse serum and/or CSF samples because ICI-associated CNS irAEs involving anti- $N$-methyl-D-aspartate receptor, anti-contactin-associated protein-like 2 or anti-Hu autoantibodies have all been reported ${ }^{95-97}$.

Myasthenia gravis or necrotizing myositis. Patients who develop myasthenia gravis during ICI treatment typically have a higher risk of myasthenic crisis than those with classical autoimmune myasthenia gravis, as well as a higher risk of myositis ${ }^{98}$. In the KEYNOTE-054 trial, in which the efficacy of adjuvant pembrolizumab was investigated in patients with resected melanoma, myositis was the only cause of treatment-related death, affecting 1 patient among $>500$ treated with pembrolizumab ${ }^{99}$ (TABLE 2). Serum creatine kinase and troponin levels should be monitored, and their elevation should raise the suspicion of myositis and/or myocarditis ${ }^{100}$. Myalgia, including ptosis, diplopia associated with limb-girdle muscular dystrophy and axial (mostly cervical) weakness are the most common symptoms. The course of disease of patients with such symptoms is typically variable, with symptom onset occurring within 6 weeks of treatment initiation (range 2-12 weeks) and with peaks of severity occurring between 1 and 4 weeks ${ }^{101}$. Antiacetylcholine receptor and myositis-associated autoantibodies are not present according to the available case reports, while the endomysial and perimysial regions of muscle biopsy samples are often densely infiltrated with macrophages and focal clusters of $\mathrm{CD} 8^{+}, \mathrm{CD}^{+}$ and $\mathrm{CD} 20^{+}$immune cells. Electroneuromyography and examinations of muscle biopsy samples are also useful methods of distinguishing between myasthenia gravis and necrotizing myositis and enable possible overlap to be documented in patients with challenging symptoms ${ }^{102}$.

GBS, GBS-like syndromes and other inflammatory neuropathies. Immune-related neuropathies occurring in patients receiving ICIs can be diffuse or focal and either acute or subacute, and patients with such symptoms can present with either motor or sensory deficits. Such neuropathies can be mild and might not require an intervention. However, severe demyelinating polyradiculoneuropathies resembling GBS have also been reported ${ }^{103}$, as have palsies affecting the cranial nerves II, VI and VII ${ }^{104-107}$. In contrast to classical GBS, ICI-induced subacute inflammatory demyelinating $\mathrm{PNP}$ is responsive to corticosteroids, in addition to the classical treatments of primary PNP (that is, intravenous immunoglobulins and plasmapheresis $)^{108}$.

\section{Ocular irAEs}

Uveitis and sicca syndrome are the main ocular irAEs of ICIs reported in the literature ${ }^{109}$. The complex mechanisms of these ocular and glandular toxicities are typically poorly understood, although various hypotheses 
have been proposed ${ }^{110}$. Patients with ICI-associated uveitis typically present with a diverse range of pathologies, which include iridocyclitis, paracentral acute middle maculopathy, retinal vasculitis, multifocal choroiditis and Vogt-Koyanagi-Harada (VKH)-like panuveitis $^{111}$. VKH is a syndrome in which classical uveitis occurs with simultaneous auditory, meningeal and skin involvement ${ }^{112}$. Sensitization to melanin-related antigens has been proposed as the aetiology of this syndrome, with PD-1-positive T cell infiltration of the uvea being involved in anterior and posterior uveitis ${ }^{113-115}$. $\mathrm{VKH}$ syndrome has been reported in patients receiving ipilimumab, pembrolizumab and in those receiving nivolumab ${ }^{116-118}$. Ulcerative keratitis, idiopathic orbital inflammation, choroidal neovascularization and melanoma-associated retinopathy have also all been described $^{119}$. The location of uveitis is variable, ranging from anterior to posterior uveitis and/or posterior sclerititis ${ }^{120}$. Comorbid painful eye movements, which are sometimes associated with chemosis and ophthalmoplegia, should raise the suspicion of ocular myositis. Data from small series of patients have revealed an association between ocular myositis and colitis, as well as between ocular myositis and thyroid disease, the latter in the absence of anti-TSH receptor autoantibodies that typically characterize the orbitopathy of Graves hyperthyroidism. The symptoms of these patients were refractory to steroids and required additional immunosuppressive drugs to obtain partial remission of the ophthalmoplegia ${ }^{121}$. Graves disease-associated orbitopathy without hyperthyroidism has also been reported, which underscores the importance of screening for impaired eye motricity and warning signs of inflammation during treatment with ICIs ${ }^{122}$.

\section{Rheumatological irAEs}

In 2017, Cappelli et al. ${ }^{123}$ published a dedicated systematic review of the literature on rheumatological and musculoskeletal irAEs. These authors reported considerable variations in the reported incidences of arthralgia and myalgia, ranging from $1 \%$ to $43 \%$ and $1 \%$ to $20 \%$, respectively ${ }^{123}$, thus highlighting the difficulties experienced by clinicians in reporting these kinds of symptoms in clinical trials. Case series and individual case reports are still the main sources of knowledge of these types of irAE and typically relate to patients with seronegative inflammatory arthritis, tenosynovitis, dermatomyositis, polymyositis and eosinophilic fasciitis. Two cases of giant cell arteritis (GCA) with polymyalgia rheumatica have been reported following treatment with ipilimumab ${ }^{124}$. A causal link was not established in the two cases of GCA in patients who received ipilimumab, although the success of abatacept (a CTLA- 4 analogue and thus a competitive antagonist) in treating patients with primary GCA underlines the hypothesis that inhibition of this immune checkpoint might increase the risk of developing overt disease.

\section{Renal irAEs}

Acute interstitial nephritis (AIN) is the most common renal irAE, with an underlying pathogenesis that differs from that of other drug-related forms of AIN, in which a delayed hypersensitivity reaction is involved as opposed to a loss of self-tolerance, as is more commonly observed in patients receiving ICIs ${ }^{125}$. Shirali et al. ${ }^{126}$ advanced the hypothesis of an alternative mechanism of ICI-induced nephritis involving the release of autoreactive $\mathrm{T}$ cells in patients receiving nephrotoxic agents, such as proton pump inhibitors and NSAIDs, owing to the high prevalence of such patients in their cohort. Once again, however, the theory of a failure of self-tolerance seems convincing because ICI-induced acute kidney injury mostly occurs in patients with a history of other irAEs. The clinical course of renal irAEs also differs from that of other drug-related AINs in that patients have a slower recovery, both after withdrawal of the causative agent and in response to corticosteroids. Patients might present with lesions consistent with glomerulonephritis (owing to the presence of immune complexes, minimal-change disease or, in one case, lupus nephritis) or even thrombotic microangiopathy (TMA) ${ }^{127}$. Patients with drug-related TMAs might not present with classical signs of haemolytic anaemia and circulating schistocytes if the thrombotic process is restricted to the renal parenchyma ${ }^{128}$.

\section{Haematological irAEs}

In contrast to other anticancer therapies, haematological irAEs in patients receiving ICIs are uncommon. Despite this rarity, a variety of manifestations have been described. Highlighting the existence of crosstalk between cellular and humoral immunity and $\mathrm{T}_{\text {reg }}$ cellmediated self-tolerance, cases of antibody-mediated haemolytic anaemia, thrombotic thrombocytopenic purpura, acquired haemophilia A, autoimmune neutropenia and autoimmune thrombocytopenia have all been reported ${ }^{129-132}$. Interestingly, cross-reactions that elicit relapsing autoimmune thrombocytopenia after sequential treatment with nivolumab and ipilimumab have been described, indicating that the same or similar irAEs might re-emerge on subsequent treatment with a different class of agent ${ }^{132}$. PD-1 expression in treatment-naive and/or memory B cells might explain the enhancement of autoreactive clones during PD-1 inhibition $^{133}$. A causative role of anti-PD-1 antibodies in $\mathrm{CD}^{+} \mathrm{T}$ cell-mediated severe bone marrow aplasia has also been suggested in a series of elderly, heavily pretreated patients ${ }^{134}$. Haemophagocytic lymphohistiocytosis is also a rare but very serious complication that is worth mentioning owing to a high mortality, which is partly attributed to diagnostic delays owing to the difficulties associated with the accurate diagnosis of this complication. Therefore, a patient presenting with severe inflammatory syndrome with associated fever, cytopenias and splenomegaly should prompt a full paraclinical work-up, including analysis of bone marrow aspirates and/or biopsy samples for the presence of haemophagocytic signs ${ }^{135}$.

\section{Management of irAEs}

Several guidelines on the management of irAEs have been published, including those provided by $\mathrm{ESMO}^{136}$, the Society for Immunotherapy of Cancer (SITC) Toxicity Management Working Group ${ }^{137}$ and the National Comprehensive Cancer Network (NCCN) ${ }^{138}$. 
These guidelines provide comprehensive general treatment algorithms for most of the frequently occurring irAEs, with clear guidelines regarding the type of immunosuppressive drugs to use and the duration of treatment based on the severity of the irAE, after ruling out other differential diagnoses (such as infectious complications, tumour progression, pulmonary embolism, cardiac events, pleural effusion and others). For this reason, we emphasize only the key general points regarding the management of irAEs and monitoring of responses to ICIs in patients receiving such treatments (BOX 1). In patients with severe and/or treatment-refractory

\section{Box $1 \mid$ Key points in the management of patients with irAEs}

- A decision to reintroduce immune-checkpoint inhibitors (ICls) following discontinuation owing to immune-related adverse events (irAEs) should be made on an individual basis, taking into account the clinical setting and specific clinical needs of each patient.

- Neurological irAEs should be managed conservatively, and rechallenge should be attempted only in patients with corticosteroid-sensitive and fully resolved peripheral neuropathies or myasthenia gravis. By contrast, patients who have had even mild encephalitis should not be re-exposed to ICls.

- Permanent discontinuation of ICls is advocated in patients with high-grade ocular, hepatic, pancreatic and/or pulmonary irAEs. Rechallenge with an anti-cytotoxic Tlymphocyte antigen 4 (CTLA-4) antibody is contraindicated owing to a high risk of relapse and/or bowel perforations in patients with severe colitis.

- A rigorous clinical examination, including an assessment of each patient's baseline bowel movements, is advised before $\mathrm{ICl}$ initiation.

- Abdominal pain, diarrhoea and/or rectal bleeding should prompt a thorough clinical work-up including the elimination of infectious causes, such as Clostridium difficile infection. Biopsy samples should be obtained for pathological description and molecular analyses.

- Grade $\geq 2-3$ colitis indicates a need to withhold ICls and start steroid therapy immediately. Infliximab should be considered in the absence of symptomatic improvements within 2-5 days. Delayed endoscopic examination is correlated with an increased risk of treatment-refractoriness ${ }^{50}$.

- Hospitalization should be considered in patients with grade $\geq 3$ irAEs and tailored regarding comorbidities, frailty status and kinetics of evolution in patients with lower-grade irAEs.

- Tapering of steroids should be considered after 48 hours of consistent symptom improvement and extended over 4-6 weeks to avoid flare phenomena related to the long half-life of ICls.

- Certain symptoms such as dyspnoea, cough and heart palpitations should advocate a full clinical work-up including the exclusion of infectious pneumonia, tumour progression, pulmonary embolism, cardiac events and pleural carcinomatosis.

- Grade 1 pneumonitis indicates a need for $\mathrm{ICl}$ withholding, with close clinical followup until resolution of symptoms. Corticosteroids should be initiated in the absence of clinical improvement. Grade $\geq 2$ disease indicates a need for corticosteroids in addition to $\mathrm{ICl}$ withholding. Infliximab and/or cyclophosphamide should be considered for refractory pneumonitis, taking into account the limited effectiveness and high risks of infection with this approach.

- A personalized immunosuppression strategy, involving monoclonal antibodies targeting key inflammatory cytokines, should be considered for patients with steroid-refractory irAEs.

- Symptom control and instauration of hormone substitution therapy (or anti-thyroid medication in patients with Graves disease) should be ensured before resuming $\mathrm{ICl}$ treatment in patients with endocrine irAEs.

- Asymptomatic biochemical abnormalities, such as elevations of serum creatinine, liver enzyme and/or troponin/creatine kinase levels, should prompt a full clinical work-up. ICls can potentially be resumed after parameter normalization, followed by close monitoring. Electrolyte disturbances should raise suspicions of endocrine irAEs (hypophysitis or adrenalitis) or renal complications.
irAEs, expert opinions regarding their management, together with the use of novel biological agents targeting key inflammatory enzymes, have been published ${ }^{139}$. Indeed, we have proposed a personalized treatment algorithm ${ }^{139}$, which is intended to guide the management of severe and/or refractory irAEs, based on the immunopathological patterns of each patient as rational tools to inform a personalized and selective immunosuppressive strategy. The predominance of the respective lymphocytic, neutrophilic and/or monocytic components present in biopsy samples obtained from affected organs could be used to guide the choice of specific immunosuppressive agents. For example, an anti-IL-6 antibody, such as tocilizumab, could be considered in patients with a $\mathrm{T}$ cell-enriched immune infiltrate, with anti-TNF antibodies, such as infliximab, reserved for those with monocytic and/or neutrophilic infiltrates. An over-representation of B cells and/or plasma cells could also be indicative of the need to add an anti-B cell therapy, such as rituximab, in patients with refractory irAEs. A proper strategy for the management of severe and/or steroid-refractory irAEs, with the upfront administration of cytokine-directed biological therapies has two main objectives: rapid inhibition of the acute phase of the inflammatory reaction, thus limiting the risk of steroid dependencies, and the inhibition of tumour development, including that promoted by cytokines such as IL-1 and IL-6. Such a rationale could provide a solution to the lack of currently validated biomarkers for the management of irAEs. This personalized algorithm was used to guide the management of a patient with severe refractory symptomatic stenosis of the upper oesophagus upon small steroid tapers, who required an 8-month course of high-dose steroids, with several bolus doses of methylprednisolone. Histological analysis of oropharynx biopsy samples revealed the presence of a predominantly $\mathrm{T}$ cellenriched infiltrate ( $\sim 90 \%$ of immune cells). Following this observation, the patient received a single intravenous dose of tocilizumab. This led to rapid amelioration of stenosis symptoms, with successful prednisone tapering without recurrent dysphagia ${ }^{140}$.

Of course, all these considerations should be discussed with the patient, taking into consideration the treatment goals and including a careful assessment of the balance between benefits and possible risks. Complex situations require a decision-making process that is coordinated across the various involved medical specialties.

\section{Autoimmune diseases}

Historically, patients with autoimmune diseases or their associated symptoms have been excluded from clinical trials involving ICIs owing to concerns regarding a higher risk of serious irAEs. However, data from three retrospective studies suggest that ICIs are generally safe and tolerable in patients with melanoma ${ }^{141,142}$ or NSCLC ${ }^{143}$ who also have autoimmune diseases. In these studies, investigators noted that only a minority of patients (23-38\%) had an exacerbation of their autoimmune disease. Furthermore, the adverse events were manageable, and permanent discontinuation of ICIs was rare. The conclusions of a systematic review of data 


\section{Box 2 | Potential risk factors for irAEs}

\section{High-risk factors (indicating preferable avoidance of immune-checkpoint} inhibitors (ICls) or, if not possible, administration of ICls under a personalized surveillance strategy)

- Connective tissue diseases (CTDs)

- Inflammatory myopathy (polymyositis and dermatomyositis), systemic lupus erythematosus, Sjögren syndrome, systemic sclerosis, antisynthetase syndrome, rheumatoid arthritis, severe psoriasis and mixed CTDs

- Vasculitis

- Granulomatosis with polyangiitis (Wegener's granulomatosis), microscopic polyangiitis, eosinophilic granulomatosis with polyangiitis (Churg-Strauss syndrome), severe Behçet disease, Takayasu arteritis, giant cell arteritis, Buerger disease, Kawasaki disease, polyarteritis nodosa, severe immunoglobulin A ( $\operatorname{lgA})$ vasculitis (Henoch-Schönlein purpura), severe cutaneous vasculitis, polymyalgia rheumatica, severe cryoglobulinaemia and undifferentiated systemic vasculitis

- Other autoimmune diseases

- Primary biliary cirrhosis, severe autoimmune hepatitis, multiple sclerosis, severe antiphospholipid syndrome, myasthenia gravis, Guillain-Barré syndrome, inflammatory bowel disease, Miller-Fisher syndrome, Vogt-Koyanagi-Harada syndrome, eosinophilic fasciitis (Shulman syndrome), relapsing polychondritis and severe autoinflammatory diseases

- Treatment-related factors

- Combination of ICls (anti-cytotoxic T lymphocyte antigen 4 (CTLA-4) antibody with either an anti-programmed cell death 1 (PD-1) or anti-programmed cell death 1 ligand 1 (PD-L1) antibody)

- Intrinsic factors

- Tumour and genetic heterogeneities, cancer type, tumour microenvironment and the microbiota

Intermediate-risk factors (administer ICls under close monitoring)

- Limited and/or previously treated autoimmune diseases

- Type I diabetes, autoimmune thyroiditis, nonsevere forms of IgA nephropathy, IgM nephropathy, Behçet disease, autoinflammatory diseases, autoimmune hepatitis and antiphospholipid syndrome, pernicious anaemia, vitiligo, Still disease and adult-onset Still disease, cold agglutinin disease, idiopathic thrombocytopenic purpura and coeliac disease

- Limited CTDs

- Psoriatic arthritis and/or psoriasis

irAE, immune-related adverse event.
Interestingly, a case report indicates that selective immunosuppression using antibodies targeting specific inflammatory mediators administered in combination with anti-PD-1 antibodies might prevent or delay the exacerbation of autoimmune diseases in patients with concurrent advanced-stage melanoma and Crohn's disease, without affecting the antitumour efficacy of the anti-PD-1 antibody ${ }^{148}$. This observation suggests that active autoimmune disease could be controlled in patients receiving anti-PD-1 antibodies using selective concomitant immunosuppression.

\section{Personalized surveillance strategies}

Clinically validated biomarkers enabling individualized assessments of the risk of irAEs are still lacking. IrAEs can occur early in the course of treatment and are sometimes associated with a severe clinical presentation. Such variations in severity suggest the presence of pre-existing factors influencing their occurrence (BOX 2). Late-onset irAEs are also difficult to predict with the available tools and, consequently, are hard to prevent. Whether or not active surveillance strategies could be useful in this setting remains a relevant question. Preventive strategies and pretreatment assessments of target organ function have long been implemented in mitigating specific chemotherapy-related toxicities. However, chemotherapy-related toxicities are more predictable than irAEs and are often related to cumulative dose and organ reserve, such as in patients receiving platinum salts and anthracyclines ${ }^{149,150}$. Severe irAEs are rare, and no evidence-based algorithms for active surveillance of such events are available, although the fact that the consequences of irAEs can be serious and even fatal in some patients is a strong argument in favour of proposing such strategies, formulated on the basis of the available data and expert opinion (BOX 3).

We believe that the establishment of formal contraindications to the use of ICIs among patients with a high risk of irAEs is not supported by strong and well-founded scientific evidence. Therefore, withholding ICIs from all patients with cancer and an autoimmune disease cannot be universally justified and could even be deleterious in light of the potentially curative effects of ICIs. Instead of imposing formal contraindications, we propose the use of a personalized risk-based surveillance strategy for each patient. An important point, which should be emphasized and explained to patients, is that the prolonged use of certain classes of immunosuppressive drugs might reduce the effectiveness of ICIs.

We propose a strategy that combines mandatory pretreatment clinical and biological assessments of all patients with an autoimmune disease, provided that such testing is locally available (BOX 3). Various risk factors for developing irAEs have been reported or suggested in the literature (BOX 2). Pre-existing autoimmune diseases, and especially those that remain active at the time of treatment onset, are examples of intrinsic risk factors with the highest available level of evidence. The use of a combination of ICIs is considered a major extrinsic risk factor. Therefore, information on patient and/or family history regarding the most frequent autoimmune symptoms should be obtained before treatment, together 


\section{Box 3 | Proposed surveillance strategy for irAEs}

\section{General pretreatment assessments}

- Performance status: including weight, height and BMI

- Cardiovascular function: including heart rate, blood pressure, electrocardiography, serum cardiac troponin and creatine kinase levels, $N$-terminal prohormone of brain natriuretic peptide (NT-proBNP), blood electrolytes and chest radiography

- Kidney function: including estimated glomerular filtration rate, urine spot analysis for proteinuria, creatininuria, calciuria, natriuria and protein to creatinine ratio

- Liver function: including total serum bilirubin, aspartate transaminase (AST), alanine transaminase (ALT), $\gamma$-glutamyl transferase (GGT) and alkaline phosphatase (ALP) levels

- Immune function and/or infection status: including serum C-reactive protein (CRP), erythrocyte sedimentation rate and complete blood counts, screening for antinuclear antibodies ${ }^{\mathrm{a}}$, complement $\mathrm{C} 3$ and/or C4 ${ }^{\mathrm{a}}$, HIV-1 or HIV-2, hepatitis B virus, hepatitis C virus and/or hepatitis E virus ${ }^{\mathrm{a}}$, human T lymphotropic virus (HTLV-1) and/or HTLV-2 ${ }^{\mathrm{a}}$ (if endemic), dosage and immunosubtraction or immunofixation of immunoglobulin $\mathrm{C}$ $(\lg G), \lg A$ and $\lg M^{a}$

- Endocrine function: including serum levels of cortisol and adrenocorticotropic hormone (ACTH) (at $8 \mathrm{am})^{\mathrm{a}}$, luteinizing hormone (LH) ${ }^{\text {a }}$, follicle-stimulating hormone $(\mathrm{FSH})^{\mathrm{a}}$, oestradiol ${ }^{\mathrm{a}}$, testosterone ${ }^{\mathrm{a}}$, thyroid-stimulating hormone $(\mathrm{TSH})$ and free T4

- Gastrointestinal function: monitoring of pretreatment bowel movements, faecal lactoferrin and calprotectin ${ }^{50}$

- Storage of pretreatment serum samples

Work-up for suspicion of specific immune-related adverse events (irAEs) and/or autoimmune diseases

- Suspected connective tissue diseases: presence of anti-Ro/SSA, anti-La/SSB, anti-Sm, anti-nRNP/U1-RNP, anti-Scl-70/anti-topoisomerase, anti-double-stranded DNA (dsDNA), anti-Jo1, anti-histone, anti-gp210, anti-p62, anti-sp100, anti-centromere and/or anti-PM-Scl autoantibodies

- Suspected vasculitis: presence of antineutrophil cytoplasmic antibodies (ANCAs) with c-ANCA proteinase, $\mathrm{p}$-ANCA myeloperoxidase and atypical ANCA ( $\mathrm{x}$-ANCA or a-ANCA) and cryoglobulinaemia

- Suspected inflammatory bowel disease: presence of anti-transglutaminase autoantibodies (anti-tTG and anti-eTG) and anti-Saccharomyces cerevisiae antibody

- Suspected autoimmune hepatitis: anti-mitochondrial, anti-liver kidney microsomal type 1 , anti-actin and anti-smooth muscle autoantibodies

- Suspected thyroiditis with anti-thyroid antibodies: anti-thyroglobulin, anti-microsomal and/or anti-thyroid peroxidase and anti-TSH receptor autoantibodies

- Suspected rheumatoid arthritis: presence of rheumatoid factor and anti-cyclic citrullinated peptides

- Suspected diabetes mellitus: presence of circulating islet cell autoantibodies

- Suspected myasthenia gravis: anti-acetylcholine receptor, anti-MUSK and anti-ganglioside antibodies

- Suspected anti-synthetase syndrome: presence of anti-phospholipid antibodies

- Suspected sarcoidosis: angiotensin-converting enzyme, corrected calcium and 24-hour calciuria measurements

\section{All patients}

- The emergence of new autoimmune disease symptoms such as arthralgia, myalgia, dyspnoea, cardiac pain or palpitation, diarrhoea, abdominal pain, sicca syndrome, cutaneous rash, conjunctivitis, scleroderma, headache, and nausea and vomiting should prompt investigations for the signs of the suspected autoimmune disease

Test is considered advisable but not mandatory.

with a careful assessment of medical history focused on autoimmunity. Other intrinsic, but less formally characterized, predisposing factors have also been suggested, such as hereditary genetic polymorphisms that increase the risk of an inflammatory response, cancer type and the nature of the immune infiltrate of the TME as well as the composition of the gut microbiota.
No clear highest-risk time window for the emergence of irAEs exists; therefore, we propose a continuous surveillance strategy for possible symptoms of autoimmune disease during ICI treatment in patients deemed to have a high risk of developing or reactivating an autoimmune disease. The kinetics of other nonspecific biological abnormalities occurring in patients with cancer, including asymptomatic increases in creatinine kinase, mild elevations in liver enzymes and serum creatinine levels, inflammatory markers and isolated autoantibodies, should be assessed carefully in order to avoid the need for unnecessary investigations ${ }^{137,151}$. Preferably, such parameters should be measured serially in the same laboratory using the same analytical methods to limit technical variability.

Isolated autoantibodies (in the absence of symptoms suggestive of autoimmune disease) are frequently detected in patients with cancer, typically owing to tumour necrosis or paraneoplasia, and are regarded as false positives in the setting of irAE management. However, the presence of such antibodies could be helpful if assessed at baseline and repeated in patients with suspected irAEs as a diagnostic adjunct by assessing the kinetics of the respective antibody titres. The interpretation of the presence of a treatment-emergent autoantibody should nevertheless be cautious in order to avoid the need for unnecessary investigations. We acknowledge that such extensive biological assessments have a financial cost and should be tailored to the availability of these tests across different cancer centres.

High-risk patients receiving ICIs should be regularly monitored for treatment-related complications by specialized multidisciplinary teams. In this context, we also emphasize the importance of having local management protocols validated by the multidisciplinary team regarding the attitude clinical staff should adopt when investigating suspected irAEs or reactivation of autoimmune diseases in patients receiving ICIs. Finally, distinguishing between either the development or reactivation of a primary autoimmune disorder and a classical irAE in patients receiving ICIs is particularly challenging, but is also highly relevant owing to the therapeutic implications.

\section{Conclusions}

The development of cancer immunotherapy will continue to shape the therapeutic landscape in the coming years, and new agents will continue to enter the clinic. New ICIs and cellular immunotherapies developed using $\mathrm{T}$ cell engineering will change the demographics and epidemiology of irAEs and reinforce the need for efficient toxicity management strategies and qualified multidisciplinary teams.

In the future, irAEs will probably be viewed as pleomorphic clinical presentations, thus posing new diagnostic and therapeutic challenges for treating physicians. Improving awareness, training a new generation of physicians with specific skills in the diagnosis and management of irAEs and encouraging multidisciplinary interactions are all essential if we are to address these emerging needs in this new and complicated area of oncology. At the same time, a conscious need exists to 
not overstate the magnitude of the problem given the low incidences of severe complications relative to those associated with more traditional cancer therapies.

Combining knowledge gathered from primary autoimmune disorders, preclinical studies, clinical trials and case series will hopefully clarify many pending questions and provide new insights into the pathophysiology of irAEs as well as reveal the optimal treatment and prevention strategies. The establishment of clinically validated early biomarkers of toxicities will also help to elaborate the most appropriate scenarios for secondary prevention that enable the resumption of ICIs following irAEs.

With regard to therapy, the most urgent unmet needs would be determining the optimal agent, dose and timing of administration for patients with steroid-refractory irAEs requiring more-selective immunosuppression. The answers to these questions are currently unclear. Other more specific needs include determining the best selective immunosuppression strategies for primary prevention of irAEs in high-risk patients without compromising the efficacy of ICIs and, similarly, determining whether or not secondary prevention strategies with selective targeting of certain cytokines would enable ICI resumption despite previous severe irAEs.

The spectrum of irAEs is broad and can potentially affect every organ with, in certain scenarios, typical irAEs that involve multiple organs. Therefore, clinicians should conduct thorough clinical and biological workups in order to minimize the risk of failing to detect silent organ and/or deferred presentations. Another goal of this Review was to raise awareness regarding the kinetics of appearance and the tendency of many irAEs to occur earlier and at a higher grade in patients receiving combinations of ICIs. Similar to most emerging challenges in clinical practice, an extensive knowledge of the characteristics of this new group of adverse events is, in our opinion, the best tool to help clinicians make the right therapeutic decisions. The introduction of a personalized surveillance strategy that enables irAEs to be managed according to the risk profile of each patient will be an important clinical development.

Published online 15 May 2019
1. Calvo, C. R., Amsen, D. \& Kruisbeek, A. M. Cytotoxic T lymphocyte antigen 4 (CTLA-4) interferes with extracellular signal-regulated kinase (ERK) and Jun NH2-terminal kinase (JNK) activation, but does not affect phosphorylation of T cell receptor zeta and ZAP70. J. Exp. Med. 186, 1645-1653 (1997).

2. Carreno, B. M. et al. CTLA-4 (CD152) can inhibit T cell activation by two different mechanisms depending on its level of cell surface expression. J. Immunol. 165, 1352-1356 (2000).

3. Takahashi, T. et al. Immunologic self-tolerance maintained by $\mathrm{CD}_{2} 5^{+} \mathrm{CD} 4^{+}$regulatory T cells constitutively expressing cytotoxic T lymphocyteassociated antigen 4. J. Exp. Med. 192, 303-310 (2000).

4. Keir, M. E., Butte, M. J., Freeman, G. J. \& Sharpe, A. H. PD-1 and its ligands in tolerance and immunity. Annu. Rev. Immunol. 26, 677-704 (2008).

5. Okazaki, T. \& Honjo, T. The PD-1-PD-L pathway in immunological tolerance. Trends Immunol. 27. 195-201 (2006)

6. Freeman, G. J. et al. Engagement of the PD-1 immunoinhibitory receptor by a novel B7 family member leads to negative regulation of lymphocyte activation. J. Exp. Med. 192, 1027-1034 (2000)

7. Schadendorf, D. et al. Pooled analysis of long-term survival data from phase II and phase III trials of ipilimumab in unresectable or metastatic melanoma. J. Clin. Oncol. 33, 1889-1894 (2015).

8. Larkin, J. et al. Combined nivolumab and ipilimumab or monotherapy in untreated melanoma. N. Engl. J. Med. 373, 23-34 (2015).

9. Motzer, R. J. et al. Nivolumab plus ipilimumab versus sunitinib in advanced renal-cell carcinoma. N. Engl. J. Med. 378, 1277-1290 (2018).

10. Overman, M. J. et al. Nivolumab in patients with metastatic DNA mismatch repair-deficient or microsatellite instability-high colorectal cancer (CheckMate 142): an open-label, multicentre, phase 2 study. Lancet Oncol. 18, 1182-1191 (2017).

11. Antonia, S. J. et al. Nivolumab alone and nivolumab plus ipilimumab in recurrent small-cell lung cancer (CheckMate 032): a multicentre, open-label, phase 1/2 trial. Lancet Oncol. 17, 883-895 (2016).

12. Hellmann, M. D. et al. Nivolumab plus ipilimumab as first-line treatment for advanced non-small-cell lung cancer (CheckMate 012): results of an open-label, phase 1, multicohort study. Lancet Oncol. 18, 31-41 (2017).

13. Hellmann, M. D. et al. Nivolumab plus ipilimumab in lung cancer with a high tumor mutational burden. N. Engl. J. Med. 378, 2093-2104 (2018).

14. Wang, D. Y. et al. Fatal toxic effects associated with immune checkpoint inhibitors: a systematic review and meta-analysis. JAMA Oncol. 4, 1721-1728 (2018).
15. Pignon, J. P. et al. Lung adjuvant cisplatin evaluation: a pooled analysis by the LACE Collaborative Group. J. Clin. Oncol. 26, 3552-3559 (2008).

16. Gooley, T. A. et al. Reduced mortality after allogeneic hematopoietic-cell transplantation. N. Engl. J. Med. 363, 2091-2101 (2010).

17. Eggermont, A. M. et al. Prolonged survival in stage III melanoma with ipilimumab adjuvant therapy. N. Engl. J. Med. 375, 1845-1855 (2016).

18. Weber, J. S., Kahler, K. C. \& Hauschild, A. Management of immune-related adverse events and kinetics of response with ipilimumab. J. Clin. Oncol. 30 2691-2697 (2012).

19. Cuzzubbo, S. et al. Neurological adverse events associated with immune checkpoint inhibitors: review of the literature. Eur. J. Cancer 73, 1-8 (2017).

20. Gelao, L., Criscitiello, C., Esposito, A., Goldhirsch, A \& Curigliano, G. Immune checkpoint blockade in cancer treatment: a double-edged sword cross-targeting the host as an "innocent bystander". Toxins (Basel) 6 , (914-933 (2014).

21. Robert, $C$. et al. Nivolumab in previously untreated melanoma without BRAF mutation. N. Engl. J. Med 372, 320-330 (2015).

22. Luis Paz-Ares, M. D. et al. Pembrolizumab plus chemotherapy for squamous non-small-cell lung cancer. N. Engl. J. Med. 379, 2040-2051 (2018).

23. Postow, M. A. et al. Nivolumab and ipilimumab versus ipilimumab in untreated melanoma. N. Engl. J. Med. 372, 2006-2017 (2015)

24. Ornstein, M. C. \& Garcia, J. A. Toxicity of checkpoint inhibition in advanced RCC: a systematic review. Kidney Cancer 1, 133-141 (2017).

25. Hammers, H. J. et al. Safety and efficacy of nivolumab in combination with ipilimumab in metastatic renal cell carcinoma: the CheckMate 016 study. J. Clin. Oncol. 35, 3851-3858 (2017)

26. Khoja, L., Day, D., Wei-Wu Chen, T., Siu, L. L. \& Hansen, A. R. Tumour- and class-specific patterns of immune-related adverse events of immune checkpoint inhibitors: a systematic review. Ann. Oncol. 28, 2377-2385 (2017).

27. El Osta, B., Hu, F., Sadek, R., Chintalapally, R. \& Tang, S. C. Not all immune-checkpoint inhibitors are created equal: meta-analysis and systematic review of immune-related adverse events in cancer trials. Crit. Rev. Oncol. Hematol. 119, 1-12 (2017).

28. Khunger, M. et al. Incidence of pneumonitis with use of programmed death 1 and programmed death-ligand 1 inhibitors in non-small cell lung cancer: a systematic review and meta-analysis of trials. Chest 152, 271-281 (2017).

29. Pillai, R. N. et al. Comparison of the toxicity profile of PD-1 versus PD-L1 inhibitors in non-small cell lung cancer: a systematic analysis of the literature. Cancer 124, 271-277 (2018).
30. Xu, C. et al. Comparative safety of immune checkpoint inhibitors in cancer: systematic review and network meta-analysis. BMJ 363, k4226 (2018).

31. Kelly, K. et al. Safety profile of avelumab in patients with advanced solid tumors: a pooled analysis of data from the phase 1 JAVELIN solid tumor and phase 2 JAVELIN Merkel 200 clinical trials. Cancer 124 2010-2017 (2018).

32. Naidoo, J. et al. Pneumonitis in patients treated with anti-programmed death-1/programmed death ligand 1 therapy. J. Clin. Oncol. 35, 709-717 (2017).

33. Yamaguchi, T et al. Pre-existing pulmonary fibrosis is a risk factor for anti-PD-1-related pneumonitis in patients with non-small cell lung cancer: a retrospective analysis. Lung Cancer 125, 212-217 (2018).

34. Kim, J. H., Kim, H. S. \& Kim, B. J. Prognostic value of smoking status in non-small-cell lung cancer patients treated with immune checkpoint inhibitors: a meta-analysis. Oncotarget 8, 93149-93155 (2017).

35. Haratani, K. et al. Association of immune-related adverse events with nivolumab efficacy in non-small-cell lung cancer. JAMA Oncol. 4, 374-378 (2018).

36. Villadolid, J. \& Amin, A. Immune checkpoint inhibitors in clinical practice: update on management of immunerelated toxicities. Transl Lung Cancer Res. 4, 560-575 (2015).

37. Belum, V. R. et al. Characterisation and management of dermatologic adverse events to agents targeting the PD-1 receptor. Eur. J. Cancer 60, 12-25 (2016).

38. Teulings, H. E. et al. Vitiligo-like depigmentation in patients with stage III-IV melanoma receiving immunotherapy and its association with survival: a systematic review and meta-analysis. J. Clin. Oncol. 33, 773-781 (2015).

39. Min Lee, C. K. et al. Characterization of dermatitis after PD-1/PD-L1 inhibitor therapy and association with multiple oncologic outcomes: a retrospective case-control study. J. Am. Acad. Dermatol. 79 1047-1052 (2018).

40. Trinidad, C. et al. Dermatologic toxicity from immune checkpoint blockade therapy with an interstitial granulomatous pattern. J. Cutan. Pathol. 45, 504-507 (2018).

41. Lacouture, M. E. et al. Ipilimumab in patients with cancer and the management of dermatologic adverse events. J. Am. Acad. Dermatol. 71, 161-169 (2014).

42. Weber, J. S. et al. Patterns of onset and resolution of immune-related adverse events of special interest with ipilimumab: detailed safety analysis from a phase 3 trial in patients with advanced melanoma. Cancer 119, 1675-1682 (2013).

43. Voudouri, D. et al. Anti-PD1/PDL1 induced psoriasis. Curr. Probl. Cancer 41, 407-412 (2017).

44. Shao, K., McGettigan, S., Elenitsas, R. \& Chu, E. Y. Lupus-like cutaneous reaction following pembrolizumab: an immune-related adverse event associated with anti-PD-1 therapy. J. Cutan. Pathol. 45, 74-77 (2018). 
45. Hwang, S. J. et al. Bullous pemphigoid, an autoantibody-mediated disease, is a novel immunerelated adverse event in patients treated with anti-programmed cell death 1 antibodies. Melanoma Res. 26, 413-416 (2016)

46. Sibaud, V. Dermatologic reactions to immune checkpoint inhibitors: skin toxicities and immunotherapy. $A m$. J. Clin. Dermatol. 19, 345-361 (2018).

47. Dougan, M. Checkpoint blockade toxicity and immune homeostasis in the gastrointestinal tract. Front. Immunol. 8, 1547 (2017).

48. Messmer, M. et al. Ipilimumab-induced enteritis without colitis: a new challenge. Case Rep. Oncol. 9, 705-713 (2016)

49. Ibraheim, H. et al. Microscopic colonic inflammation in immune check point inhibitor-induced diarrhoea/ colitis [abstract PWE-025]. Gut 67 (Suppl. 1), A80 (2018).

50. Abu-Sbeih, H. et al. Importance of endoscopic and histological evaluation in the management of immune checkpoint inhibitor-induced colitis. J. Immunother. Cancer 6, 95 (2018).

51. Johnson, D. H. et al. Infliximab associated with faster symptom resolution compared with corticosteroids alone for the management of immune-related enterocolitis. J. Immunother. Cancer 6, 103 (2018)

52. Read, S., Malmstrom, V. \& Powrie, F. Cytotoxic T lymphocyte-associated antigen 4 plays an essential role in the function of $\mathrm{CD} 25^{+} \mathrm{CD} 4^{+}$regulatory cells that control intestinal inflammation. J. Exp. Med 192, 295-302 (2000).

53. Zeissig, S. et al. Early-onset Crohn's disease and autoimmunity associated with a variant in CTLA-4. Gut 64, 1889-1897 (2015)

54. Pitt, J. M. et al. Enhancing the clinical coverage and anticancer efficacy of immune checkpoint blockade through manipulation of the gut microbiota. Oncoimmunology 6, e1132137 (2017).

55. Vetizou, M. et al. Anticancer immunotherapy by CTLA-4 blockade relies on the gut microbiota. Science 350, 1079-1084 (2015).

56. Chaput, N. et al. Baseline gut microbiota predicts clinical response and colitis in metastatic melanoma patients treated with ipilimumab. Ann. Oncol. 28, 1368-1379 (2017)

57. Dubin, K. et al. Intestinal microbiome analyses identify melanoma patients at risk for checkpointblockade-induced colitis. Nat. Commun. 7, 10391 (2016).

58. Wang, Y. et al. Fecal microbiota transplantation for refractory immune checkpoint inhibitor-associated colitis. Nat. Med. 24, 1804-1808 (2018)

59. Tarhini, A. A. et al. Baseline circulating IL-17 predicts toxicity while TGF-beta 1 and IL-10 are prognostic of relapse in ipilimumab neoadjuvant therapy of melanoma. J. Immunother. Cancer 3, 39 (2015).

60. Kanai, T. et al. Blockade of B7-H1 suppresses the development of chronic intestinal inflammation. J. Immunol. 171, 4156-4163 (2003).

61. Reynoso, E. D. et al. Intestinal tolerance is converted to autoimmune enteritis upon PD-1 ligand blockade. J. Immunol. 182, 2102-2112 (2009).

62. Chuzi, S. et al. Clinical features, diagnostic challenges, and management strategies in checkpoint inhibitorrelated pneumonitis. Cancer Manag. Res. 9, 207-213 (2017).

63. Nishino, M., Giobbie-Hurder, A., Hatabu, H. Ramaiya, N. H. \& Hodi, F. S. Incidence of programmed cell death 1 inhibitor-related pneumonitis in patients with advanced cancer: a systematic review and meta-analysis. JAMA Oncol. 2, 1607-1616 (2016).

64. Koelzer, V. H. et al. Systemic inflammation in a melanoma patient treated with immune checkpoint inhibitors-an autopsy study. J. Immunother. Cancer 4, 13 (2016)

65. Leroy, V. et al. Pembrolizumab-induced pneumonitis. ERJ Open Res. https://doi.org/10.1183/23120541. 00081-2016 (2017).

66. Montaudie, H., Pradelli, J., Passeron, T., Lacour, J. P. \& Leroy, S. Pulmonary sarcoid-like granulomatosis induced by nivolumab. Br. J. Dermatol. 176 1060-1063 (2017).

67. Cukier, P., Santini, F. C., Scaranti, M. \& Hoff, A. O. Endocrine side effects of cancer immunotherapy. Endocr. Relat. Cancer 24, T331-T347 (2017).

68. Faje, A. Immunotherapy and hypophysitis: clinical presentation, treatment, and biologic insights. Pituitary 19, 82-92 (2016).

69. Blansfield, J. A. et al. Cytotoxic T-lymphocyte-associated antigen- 4 blockage can induce autoimmune hypophysitis in patients with metastatic melanoma and renal cancer. J. Immunother. 28, 593-598 (2005).
70. Corsello, S. M. et al. Endocrine side effects induced by immune checkpoint inhibitors. J. Clin. Endocrinol. Metab. 98, 1361-1375 (2013).

71. Iwama, S. et al. Pituitary expression of CTLA-4 mediates hypophysitis secondary to administration of CTLA-4 blocking antibody. Sci. Transl Med. 6 , 230ra245 (2014).

72. Caturegli, P. et al. Hypophysitis secondary to cytotoxic T-lymphocyte-associated protein 4 blockade: insights into pathogenesis from an autopsy series. Am. J. Pathol. 186, 3225-3235 (2016).

73. Ribas, A. et al. Phase III randomized clinical trial comparing tremelimumab with standard-of-care chemotherapy in patients with advanced melanoma J. Clin. Oncol. 31, 616-622 (2013)

74. Okano, Y. et al. Nivolumab-induced hypophysitis in a patient with advanced malignant melanoma. Endocr. J. 63, 905-912 (2016).

75. Gonzalez-Rodriguez, E. \& Rodriguez-Abreu, D. \& Spanish Group for Cancer Immuno-Biotherapy (GETICA). Immune checkpoint inhibitors: review and management of endocrine adverse events. Oncologist 21, 804-816 (2016).

76. de Filette, J. et al. Incidence of thyroid-related adverse events in melanoma patients treated with pembrolizumab. J. Clin. Endocrinol. Metab. 101, 4431-4439 (2016).

77. Lee, $\mathrm{H}$. et al. Characterization of thyroid disorders in patients receiving immune checkpoint inhibition therapy. Cancer Immunol. Res. 5, 1133-1140 (2017).

78. Yamauchi, I. et al. Clinical features of nivolumab-induced thyroiditis: a case series study. Thyroid 27, 894-901 (2017).

79. Robert, C. et al. Pembrolizumab versus ipilimumab in advanced melanoma. N. Engl. J. Med. 372 2521-2532 (2015)

80. Wang, W., Lie, P., Guo, M. \& He, J. Risk of hepatotoxicity in cancer patients treated with immune checkpoint inhibitors: A systematic review and meta-analysis of published data. Int. J. Cancer 141, 1018-1028 (2017).

81. Suzman, D. L., Pelosof, L., Rosenberg A \& Avigan, M. Hepatotoxicity of immune checkpoint inhibitors: an evolving picture of risk associated with a vital class of immunotherapy agents. Liver Int. 38, 976-987 (2018).

82. Karamchandani, D. M. \& Chetty, R. Immune checkpoint inhibitor-induced gastrointestinal and hepatic injury: pathologists' perspective. J. Clin. Pathol. 71, 665-671 (2018)

83. Johncilla, M. et al. Ipilimumab-associated hepatitis: clinicopathologic characterization in a series of 11 cases. Am. J. Surg. Pathol. 39, 1075-1084 (2015).

84. Everett, J., Srivastava, A. \& Misdraji, J. Fibrin ring granulomas in checkpoint inhibitor-induced hepatitis. Am. J. Surg. Pathol. 41, 134-137 (2017).

85. Mahmood, S. S. et al. Myocarditis in patients treated with immune checkpoint inhibitors. J. Am. Coll. Cardiol. 71, 1755-1764 (2018)

86. Johnson, D. B. et al. Fulminant myocarditis with combination immune checkpoint blockade. N. Engl. J. Med. 375, 1749-1755 (2016).

87. Moslehi, J. J., Salem, J. E., Sosman, J. A., Lebrun-Vignes, B. \& Johnson, D. B. Increased reporting of fatal immune checkpoint inhibitor-associated myocarditis. Lancet 391, 933 (2018).

88. Heinzerling, L. et al. Cardiotoxicity associated with CTLA4 and PD1 blocking immunotherapy. J. Immunother. Cancer 4, 50 (2016).

89. Varricchi, G. et al. Cardiotoxicity of immune checkpoint inhibitors. ESMO Open 2, e000247 (2017).

90. Matson, D. R., Accola, M. A., Rehrauer, W. M. \& Corliss, R. F. Fatal myocarditis following treatment with the PD-1 inhibitor nivolumab. J. Forensic Sci. 63 954-957 (2017)

91. Touat, M., Talmasov, D., Ricard, D. \& Psimaras, D Neurological toxicities associated with immunecheckpoint inhibitors. Curr. Opin. Neurol. 30, 659-668 (2017).

92. Kao, J. C. et al. Neurological complications associated with anti-programmed death 1 (PD-1) antibodies. JAMA Neurol. 74, 1216-1222 (2017).

93. Astaras, C., de Micheli, R., Moura, B., Hundsberger, T. \& Hottinger, A. F. Neurological adverse events associated with immune checkpoint inhibitors: diagnosis and management. Curr. Neurol. Neurosci. Rep. 18, 3 (2018).

94. Ghosn, J. et al. A severe case of neuro-Sjogren's syndrome induced by pembrolizumab. J. Immunother. Cancer 6, 110 (2018)

95. Williams, T. J. et al. Association of autoimmune encephalitis with combined immune checkpoint inhibitor treatment for metastatic cancer. JAMA Neurol. 73, 928-933 (2016).
96. Brown, M. P., Hissaria, P., Hsieh, A. H., Kneebone, C. \& Vallat, W. Autoimmune limbic encephalitis with anticontactin-associated protein-like 2 antibody secondary to pembrolizumab therapy. J. Neuroimmunol. $\mathbf{3 0 5}$, 16-18 (2017)

97. Papadopoulos, K. P. et al. Anti-Hu-associated autoimmune limbic encephalitis in a patient with PD-1 inhibitor-responsive myxoid chondrosarcoma. Oncologist 23, 118-120 (2018).

98. Chen, J. H., Lee, K. Y., Hu, C. J. \& Chung, C. C. Coexisting myasthenia gravis, myositis, and polyneuropathy induced by ipilimumab and nivolumab in a patient with non-small-cell lung cancer: a case report and literature review. Medicine (Baltimore) 96 e9262 (2017).

99. Eggermont, A. M. M. et al. Adjuvant pembrolizumab versus placebo in resected stage III melanoma. N. Engl. J. Med. 378, 1789-1801 (2018).

100. Suzuki, S. et al. Nivolumab-related myasthenia gravis with myositis and myocarditis in Japan. Neurology 89 1127-1134 (2017)

101. Makarious et al. Myasthenia gravis: an emerging toxicity of immune checkpoint inhibitors. Eur. J. Cancer 82, 128-136 (2017)

102. Huh, S. Y. et al. Emergence of myasthenia gravis with myositis in a patient treated with pembrolizumab for thymic cancer. J. Clin Neurol. 14, 115-117 (2018).

103. Butt, S. \& Bhaumik, S. Acute demyelinating polyneuropathy caused by nivolumab in a man with metastatic non-small cell lung cancer. J. Gerontol. Geriatr. Res. 5, 302 (2016).

104. Johnson, D. B. et al. Survivorship in immune therapy: assessing chronic immune toxicities, health outcomes, and functional status among long-term ipilimumab survivors at a single referral center. Cancer Immunol. Res. 3, 464-469 (2015)

105. Yeh, O. L \& Francis, C. E. Ipilimumab-associated bilateral optic neuropathy. J. Neuroophthalmol 35, 144-147 (2015)

106. Altman, A. L., Golub, J. S., Pensak, M. L. \& Samy, R. N. Bilateral facial palsy following ipilimumab infusion for melanoma. Otolaryngol. Head Neck Surg. 153, 894-895 (2015).

107. Voskens, C. J. et al. The price of tumor control: an analysis of rare side effects of anti-CTLA- 4 therapy in metastatic melanoma from the ipilimumab network. PLOS ONE 8, e53745 (2013).

108. Fukumoto, Y., Kuwahara, M., Kawai, S., Nakahama, K. $\&$ Kusunoki, S. Acute demyelinating polyneuropathy induced by nivolumab. J. Neurol. Neurosurg. Psychiatry 89, 435-437 (2018).

109. Abdel-Rahman, O. et al. Immune-related ocular toxicities in solid tumor patients treated with immune checkpoint inhibitors: a systematic review. Expert Rev. Anticancer Ther. 17, 387-394 (2017).

110. Caspi, R. R. Immunotherapy of autoimmunity and cancer: the penalty for success. Nat. Rev. Immunol. 8 970-976 (2008).

111. Conrady, C. D. et al. Checkpoint inhibitor-induced uveitis: a case series. Graefes Arch. Clin. Exp. Ophthalmol. 256, 187-191 (2018).

112. Read, R. W. et al. Complications and prognostic factors in Vogt-Koyanagi-Harada disease. Am. J. Ophthalmol. 131, 599-606 (2001).

113. Caspi, R. R. A look at autoimmunity and inflammation in the eye. J. Clin. Invest. 120, 3073-3083 (2010).

114. Broekhuyse, R. M., Kuhlmann, E. D. \& Winkens, H. J. Experimental autoimmune anterior uveitis (EAAU). III. Induction by immunization with purified uveal and skin melanins. Exp. Eye Res. 56, 575-583 (1993).

115. Chen, L. et al. Constitutive neuronal expression of the immune regulator, programmed death 1 (PD-1) identified during experimental autoimmune uveitis. Ocul. Immunol. Inflamm. 17, 47-55 (2009).

116. Matsuo, T. \& Yamasaki, O. Vogt-Koyanagi-Harada disease-like posterior uveitis in the course of nivolumab (anti-PD-1 antibody), interposed by vemurafenib (BRAF inhibitor), for metastatic cutaneous malignan melanoma. Clin. Case Rep. 5, 694-700 (2017).

117. Tamura, T. et al. Vogt-Koyanagi-Harada syndrome induced by pembrolizumab in a patient with non-smallcell lung cancer. J. Thorac Oncol. 13, 1606-1607 (2018).

118. Crosson, J. N. et al. Vogt-Koyanagi-Harada-like syndrome after CTLA-4 inhibition with ipilimumab for metastatic melanoma. J. Immunother. 38, 80-84 (2015).

119. Antoun, J., Titah, C. \& Cochereau, I. Ocular and orbital side-effects of checkpoint inhibitors: a review article. Curr. Opin. Oncol. 28, 288-294 (2016).

120. Gonzales, J. A., Shantha, J. \& Acharya, N. R. Combination nivolumab- and cabiralizumab-associated 
acute bilateral anterior and posterior scleritis and anterior uveitis. Am. J. Ophthalmol Case Rep. 10 117-118 (2018)

121. Pushkarevskaya, A., Neuberger, U., Dimitrakopoulou-Strauss, A., Enk, A. \& Hassel, J. C. Severe ocular myositis after ipilimumab treatment for melanoma: a report of 2 cases. J. Immunother. 40 , 282-285 (2017)

122. Campredon, P. et al. Severe inflammatory ophthalmopathy in a euthyroid patient during nivolumab treatment. Eur. Thyroid J 7, 84-87 (2018).

123. Cappelli, L. C., Gutierrez, A. K., Bingham, C. O. 3rd $\Sigma$ Shah, A. A. Rheumatic and musculoskeletal immune related adverse events due to immune checkpoint inhibitors: a systematic review of the literature. Arthritis Care Res. (Hoboken) 69, 1751-1763 (2017).

124. Goldstein, B. L., Gedmintas, L. \& Todd, D. J. Drug-associated polymyalgia rheumatica/giant cell arteritis occurring in two patients after treatment with ipilimumab, an antagonist of ctla-4. Arthritis Rheumatol. 66, 768-769 (2014).

125. Vanoverbeke, L. $\&$ Sprangers, B. Management of checkpoint inhibitor-associated renal toxicities. Expert Rev. Qual. Life Cancer Care 2, 215-223 (2017).

126. Shirali, A. C., Perazella, M. A. \& Gettinger, S. Association of acute interstitial nephritis with programmed cell death 1 inhibitor therapy in lung cancer patients. $A m$. J. Kidney Dis. 68, 287-291 (2016).

127. Cortazar, F. B. et al. Clinicopathological features of acute kidney injury associated with immune checkpoint inhibitors. Kidney Int. 90, 638-647 (2016).

128. Izzedine, H. \& Perazella, M. A. Anticancer drug induced acute kidney injury. Kidney Int. Rep. 2 504-514 (2017).

129. Kong B. Y., Micklethwaite, K. P. Swaminathan, S Kefford, R. F. \& Carlino, M. S. Autoimmune hemolytic anemia induced by anti-PD-1 therapy in metastatic melanoma. Melanoma Res. 26, 202-204 (2016)

130. King, J., de la Cruz, J. \& Lutzky, J. Ipilimumab-induced thrombotic thrombocytopenic purpura (TTP). J. Immunother. Cancer 5, 19 (2017)

131. Delyon, J., Mateus, C. \& Lambert, T. Hemophilia A induced by ipilimumab. N. Engl. J. Med. 365 1747-1748 (2011).

132. Shiuan, E. et al. Thrombocytopenia in patients with melanoma receiving immune checkpoint inhibitor therapy. J. Immunother. Cancer 5, 8 (2017).

133. Thibult, M. L. et al. PD-1 is a novel regulator of human B cell activation. Int. Immunol. 25, 129-137 (2013)

134. Michot, J. M. et al. Immune-related bone marrow failure following anti-PD1 therapy. Eur. J. Cancer $\mathbf{8 0}$ 1-4 (2017).

135. Sadaat, M. \& Jang, S. Hemophagocytic lymphohistiocytosis with immunotherapy: brief review and case report. J. Immunother. Cancer 6, 49 (2018).

136. Haanen, J. et al. Management of toxicities from immunotherapy: ESMO Clinical Practice Guidelines for diagnosis, treatment and follow-up. Ann. Oncol. 28, (Suppl. 4), iv119-iv142 (2017).

137. Puzanov I et al. Managing toxicities associated with immune checkpoint inhibitors: consensus recommendations from the Society for Immunotherapy of Cancer (SITC) Toxicity Management Working Group. J. Immunother. Cancer 5, 95 (2017).

138. National Comprehensive Cancer Network. Management of immunotherapy-related toxicities. NCCN https:// www.nccn.org/professionals/physician_gls/pdf/ immunotherapy.pdf (2018)

139. Martins, F. et al. New therapeutic perspectives to manage refractory immune checkpoint-related toxicities Lancet Oncol 20, e54-e64 (2019).

140. Horisberger, A. et al. A severe case of refractory esophageal stenosis induced by nivolumab and responding to tocilizumab therapy. J. Immunother. Cancer 6, 156 (2018).
141. Johnson, D. B. et al. Ipilimumab therapy in patients with advanced melanoma and preexisting autoimmune disorders. JAMA Oncol. 2, 234-240 (2016).

142. Menzies, A. M. et al. Anti-PD-1 therapy in patients with advanced melanoma and preexisting autoimmune disorders or major toxicity with ipilimumab. Ann. Oncol. 28, 368-376 (2017).

143. Leonardi, G. C. et al. Safety of programmed death-1 pathway inhibitors among patients with non-small-cell lung cancer and preexisting autoimmune disorders. J. Clin. Oncol. 36, 1905-1912 (2018).

144. Abdel-Wahab, N., Shah, M., Lopez-Olivo, M. A \& Suarez-Almazor, M. E. Use of immune checkpoint inhibitors in the treatment of patients with cancer and preexisting autoimmune disease. Ann. Intern. Med. 169, 133-134 (2018).

145. Brahmer, J. et al. Nivolumab versus docetaxel in advanced squamous-cell non-small-cell lung cancer. N. Engl. J. Med. 373, 123-135 (2015)

146. Herbst, R. S. et al. Pembrolizumab versus docetaxel for previously treated, PD-L1-positive, advanced nonsmall-cell lung cancer (KEYNOTE-010): a randomised controlled trial. Lancet 387, 1540-1550 (2016).

147. Reck, M. et al. Pembrolizumab versus chemotherapy for PD-L1-positive non-small-cell lung cancer. N. Engl. J. Med. 375, 1823-1833 (2016).

148. Uemura, M. et al. Selective inhibition of autoimmune exacerbation while preserving the anti-tumor clinical benefit using IL- 6 blockade in a patient with advanced melanoma and Crohn's disease: a case report. J. Hematol. Oncol. 9, 81 (2016).

149. Volkova, M. \& Russell, R. 3rd. Anthracycline cardiotoxicity: prevalence, pathogenesis and treatment. Curr. Cardiol. Rev. 7, 214-220 (2011)

150. Callejo, A., Sedó-Cabezón, L., Juan, I. D. \& Llorens, J. Cisplatin-induced ototoxicity: effects, mechanism and protection strategies. Toxics 3, 268-293 (2015).

151. Solans-Laque, R. et al. Clinical significance of antinuclear antibodies in malignant diseases: association with rheumatic and connective tissue paraneoplastic syndromes. Lupus 13, 159-164 (2004).

152. Collins, L. K., Chapman, M. S., Carter, J. B. \& Samie, F. H. Cutaneous adverse effects of the immune checkpoint inhibitors. Curr Probl. Cancer 41, 125-128 (2017).

153. Wang, P. F. et al. Immune-related adverse events associated with anti-PD-1/PD-L1 treatment for malignancies: a meta-analysis. Front. Pharmacol. 8, 730 (2017).

154. Bertrand, A., Kostine, M., Barnetche, T., Truchetet, M. E. $\&$ Schaeverbeke, T. Immune related adverse events associated with anti-CTLA-4 antibodies: systematic review and meta-analysis. BMC Med. 13, 211 (2015).

155. Spain, L. \& Larkin, J. Combination immune checkpoint blockade with ipilimumab and nivolumab in the management of advanced melanoma. Expert Opin. Biol. Ther. 16, 389-396 (2016).

156. Soularue, E. et al. Enterocolitis due to immune checkpoint inhibitors: a systematic review. Gut 67 2056-2067 (2018).

157. Beck, K. E. et al. Enterocolitis in patients with cance after antibody blockade of cytotoxic T-lymphocyteassociated antigen 4. J. Clin. Oncol. 24, 2283-2289 (2006).

158. Gupta, A., De Felice, K. M., Loftus, E. V. Jr \& Khanna, S Systematic review: colitis associated with anti-CTLA-4 therapy. Aliment. Pharmacol. Ther. 42, 406-417 (2015).

159. Girotra, M. et al. The current understanding of the endocrine effects from immune checkpoint inhibitors and recommendations for management. J NCl Cancer Spectr. 2, pky021 (2018)

160. Faje, A. T. et al. Ipilimumab-induced hypophysitis: a detailed longitudinal analysis in a large cohort of patients with metastatic melanoma. J. Clin. Endocrinol. Metab. 99, 4078-4085 (2014).
161. Patel, N. S., Oury, A., Daniels, G. A., Bazhenova, L. \& Patel, S. P. Incidence of thyroid function test abnormalities in patients receiving immune-checkpoint inhibitors for cancer treatment. Oncologist 23 1236-1241 (2018).

162. De Martin, E. et al. Characterization of liver injury induced by cancer immunotherapy using immune checkpoint inhibitors. J. Hepatol. 68, 1181-1190 (2018).

163. Gauci, M. L. et al. Immune-related hepatitis with immunotherapy: are corticosteroids always needed? J. Hepatol. 69, 548-550 (2018).

164. Wanchoo, R. et al. Adverse renal effects of immune checkpoint inhibitors: a narrative review. Am. J. Nephrol. 45, 160-169 (2017).

165. Calabrese, C., Kirchner, E., Kontzias, K., Velcheti, V. \& Calabrese, L. H. Rheumatic immune-related adverse events of checkpoint therapy for cancer: case series of a new nosological entity. RMD Open 3, e000412 (2017).

166. Hodi, F. S. et al. Improved survival with ipilimumab in patients with metastatic melanoma. N. Engl. J. Med. 363, 711-723 (2010).

167. Borghaei, H. et al. Nivolumab versus docetaxel in advanced nonsquamous non-small-cell lung cancer N. Engl. J. Med. 373, 1627-1639 (2015)

168. Wolchok, J. D. et al Overall survival with combined nivolumab and ipilimumab in advanced melanoma. N. Engl. J. Med. 377, 1345-1356 (2017).

169. Gulley, J. L. et al. Avelumab for patients with previously treated metastatic or recurrent non-small-cell lung cancer (JAVELIN Solid Tumor): dose-expansion cohort of a multicentre, open-label, phase $1 \mathrm{~b}$ trial. Lancet Oncol. 18, 599-610 (2017).

170. Kaufman, H. L. et al. Avelumab in patients with chemotherapy-refractory metastatic Merkel cell carcinoma: a multicentre, single-group, open-label, phase 2 trial. Lancet Oncol. 17, 1374-1385 (2016).

171. Rittmeyer, A. et al. Atezolizumab versus docetaxel in patients with previously treated non-small-cell lung cancer (OAK): a phase 3, open-label, multicentre randomised controlled trial. Lancet 389, 255-265 (2017).

172. Garassino, M. C. et al. Durvalumab as third-line or later treatment for advanced non-small-cell lung cancer (ATLANTIC): an open-label, single-arm, phase 2 study. Lancet Oncol. 19, 521-536 (2018).

\section{Acknowledgements}

F.M. acknowledges the support of the Personalized Health and Related Technologies (PHRT) strategic focus area of the Swiss Federal Institutes of Technology (ETH) Domain and the Swiss Personalized Health Network (SPHN) initiative of the Swiss Academy of Medical Sciences (project no. 2017407). The work of G.P.S. is partly supported by a 2016 Leenaards Foundation Fellowship for Academic Advancement in Clinical Medicine. The authors thank R. Guindon for his help in the conception of the figures.

\section{Author contributions}

F.M. and M.O. researched data for this manuscript. F.M. J.A.T. and M.O. discussed manuscript content. All authors wrote the manuscript. F.M. G.P.S and M.O. edited and/or reviewed the manuscript before submission.

\section{Competing interests}

S.P. has received education grants from and provided consultation, attended advisory boards and/or provided lectures for the following organizations: Amgen, AstraZeneca, Boehringer-Ingelheim, Bristol-Myers Squibb, Clovis, Eli Lilly, F. Hoffmann-La Roche, Janssen, Merck Serono, Merck Sharp and Dohme, Novartis, Pfizer, Regeneron and Takeda. All other authors declare no competing interests.

\section{Publisher's note}

Springer Nature remains neutral with regard to jurisdictional claims in published maps and institutional affiliations. 